\title{
Helical vortices: quasi-equilibrium states and their time evolution
}

\author{
Can Selçuk \\ LIMSI, CNRS, Université Paris-Saclay, rue John von Neumann, 91405 Orsay, France and \\ Sorbonne Universités, UPMC Univ Paris 06, IFD, 4 place Jussieu, 75005 Paris, France \\ Ivan Delbende \\ Sorbonne Universités, UPMC Univ Paris 06, UFR d'Ingénierie, 4 place Jussieu, 75005 Paris, France and \\ LIMSI, CNRS, Université Paris-Saclay, rue John von Neumann, 91405 Orsay, France \\ Maurice Rossi \\ CNRS, UMR 7190, Institut Jean Le Rond d'Alembert, 75005 Paris, France and \\ Sorbonne Universités, UPMC Univ Paris 06, UMR 7190, \\ Institut Jean Le Rond d'Alembert, 75005 Paris, France
}

(Dated: July 25, 2017)

The time evolution of a viscous helical vortex is investigated by direct numerical simulations of the NavierStokes equations where helical symmetry is enforced. Using conservation laws in the framework of helical symmetry, we elaborate an initial condition consisting in a finite core vortex, the time evolution of which leads to a generic quasi-equilibrium state independent of the initial core size. Numerical results at different helical pitch values provide an accurate characterization in time for such helical states, for which specific techniques have been introduced: helix radius, angular velocity, streamfunction/velocity/vorticity relationships, core properties (size, self-similarity and ellipticity). Viscosity is shown to be at the origin of a small helical velocity component which we relate to the helical vorticity component. Finally, changes in time of the flow topology are studied using the helical streamfunction and three-dimensional Lagrangian orbits. 


\section{INTRODUCTION}

Flows behind rotating bladed devices are often dominated by interlaced helical vortices developing from blade tips. Such vortex systems are commonly found in the wake of marine propellers, wind turbines or helicopters. Important issues such as noise reduction, helicopter descent flight safety or wind farm optimization have generated a large amount of studies aiming at predicting vortex emission, jet or wake spreading, instability growth and turbulent mixing in this context. Experiments, as well as some numerical works, investigate the production of the wake by the rotor blades, the spatial three-dimensional evolution and instability growth [1-10]. By contrast, most theoretical studies adopt a local approach where the underlying vortex system is assumed to be purely helical: this amounts to consider the flow in a cut plane orthogonal to the helix axis and assume invariance by translating and rotating the solution along that axis to yield the complete three-dimensional flow. In this framework, equilibrium states have been investigated [11-15], as well as their instabilities [16-22]. Among the studies devoted to equilibria, some concern thin-core helical vortices where the main task is to remove the singular behaviour of curved filaments to compute the self-induced velocities and deduce, for instance, the angular velocity of the full vortex system, or the topology of streamlines [23]. Vortices with arbitrary core sizes have been numerically investigated by Lucas \& Dritschel [24]: using the helically symmetric Euler equations for a given helical pitch, they determine patch vortex solutions steady in some rotating frame, imposing geometrical constraints on the centroid location and the vortex core size. The goal of the present paper is to extend the study of helically symmetric vortices of arbitrary core size to the viscous context.

In a recent study [25], the internal structure of helical vortex configurations has been determined using asymptotic analysis. A viscous axisymmetric vortex core structure of the Batchelor-vortex type was assumed at leading order; the dipolar (core shift) and quadrupolar corrections (elliptical deformation) due to local curvature and nonlocal external strain were then quantified. Such theory successfully described helical vortex states when compared to numerical results obtained using a Navier-Stokes solver with built-in helical symmetry [26]. In the present work, we use this built-in helical symmetry solver to compute the time evolution of a helical vortex with different helical pitches. We thus bring additional information, namely the velocity/vorticity profiles selected by viscous effects and their time evolution. Some of these aspects were already considered for systems of two [27] or three [28] helical vortices. We herein focus on the case of one single helical vortex in quasi-equilibrium state, and investigate the relationships between streamfunction, helical velocity and vorticity, as well as the vortex core properties (size, self-similarity and ellipticity) and flow topology.

The paper is structured as follows: in section II, the concept of helical symmetric flow is recalled together with the equations governing such flows. The numerical implementation as well as the vortex characterization method are also briefly provided. In section III, conservation laws for helical vortices in the inviscid and viscous frameworks are given. The rest of the paper is more specific to the study of a single helical vortex: section IV focuses on initial conditions and describes the relaxation towards a generic quasi-equilibrium state. The characterization of the quasi-equilibrium itself is accounted for in section $\mathrm{V}$. The original methodology used for vortex characterization is presented in detail in the appendices.

\section{HELICAL SYMMETRIC FLOWS}

The flows presented here all display a helical symmetry of helical pitch $2 \pi L$ ( $L$ is hereafter called the reduced pitch): they are invariant through any combined translation of length $\Delta$ along the $z$-axis and rotation of angle $\Delta / L$ about the same axis. In standard cylindrical coordinates $(r, \theta, z)$, scalar (resp. vector) fields are helically symmetric if these scalar fields (resp. the cylindrical components of these vectors) depend on space only through $r$ and $\varphi \equiv \theta-z / L$. For instance, the helically symmetric velocity field $\boldsymbol{u}(r, \theta, z, t)$ can be expressed 


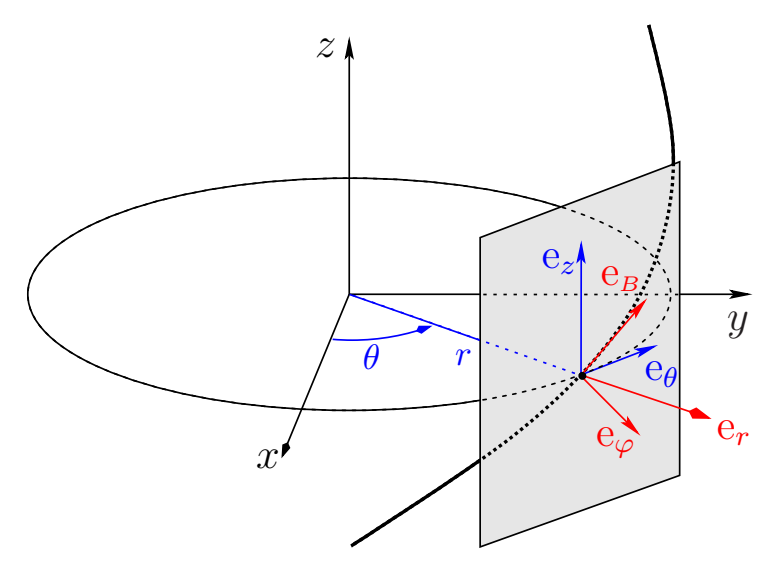

FIG. 1: Local polar (blue) and helical (red) basis.

as follows:

$$
\boldsymbol{u}=u_{r}(r, \varphi, t) \boldsymbol{e}_{r}(\theta)+u_{\theta}(r, \varphi, t) \boldsymbol{e}_{\theta}(\theta)+u_{z}(r, \varphi, t) \boldsymbol{e}_{z} .
$$

It is also convenient to introduce a local orthonormal Serret-Frénet basis related to helical lines i.e. lines of constant $\varphi$ (figure 1). This basis $\left(\boldsymbol{e}_{B}, \boldsymbol{e}_{r}, \boldsymbol{e}_{\varphi}\right)$ is defined by

$$
\boldsymbol{e}_{B}(r, \theta)=\alpha(r)\left[\boldsymbol{e}_{z}+\frac{r}{L} \boldsymbol{e}_{\theta}(\theta)\right], \boldsymbol{e}_{\varphi}(r, \theta)=\alpha(r)\left[\boldsymbol{e}_{\theta}(\theta)-\frac{r}{L} \boldsymbol{e}_{z}\right],
$$

with a normalisation factor $\alpha(r)=\left(1+r^{2} / L^{2}\right)^{-1 / 2} \leq 1$. In this basis, the velocity field is decomposed as:

$$
\boldsymbol{u}=u_{r}(r, \varphi, t) \boldsymbol{e}_{r}(\theta)+u_{\varphi}(r, \varphi, t) \boldsymbol{e}_{\varphi}(r, \theta)+u_{B}(r, \varphi, t) \boldsymbol{e}_{B}(r, \theta) .
$$

Such decomposition is also applied on the vorticity field $\boldsymbol{\omega}$.

\section{A. Governing equations for helically symmetric flows}

For helically symmetric flows, the incompressible Navier-Stokes equations can be rewritten in a way that directly takes into account this symmetry. The divergenceless character of both velocity and vorticity is automatically taken care of by introducing the helical component of velocity $u_{B}(r, \varphi, t)$, of vorticity $\omega_{B}(r, \varphi, t)$ and a streamfunction $\Psi(r, \varphi, t)$ such that [24]

$$
\begin{gathered}
\boldsymbol{u}=u_{B}(r, \varphi, t) \boldsymbol{e}_{B}+\alpha(r) \nabla \Psi(r, \varphi, t) \times \boldsymbol{e}_{B}, \\
\boldsymbol{\omega}=\omega_{B}(r, \varphi, t) \boldsymbol{e}_{B}+\alpha(r) \nabla\left(\frac{u_{B}(r, \varphi, t)}{\alpha(r)}\right) \times \boldsymbol{e}_{B} .
\end{gathered}
$$


Fields $u_{B}, \omega_{B}$ and $\Psi$ are related via a generalisation of the two-dimensional $\Psi-\omega$ relationship in the helical context:

$$
\mathbb{L} \Psi=-\omega_{B}+\frac{2 \alpha^{2}}{L} u_{B},
$$

where $\mathbb{L}$ stands for the modified Laplace operator

$$
\mathbb{L}(\cdot)=\frac{1}{r \alpha} \frac{\partial}{\partial r}\left(r \alpha^{2} \frac{\partial(\cdot)}{\partial r}\right)+\frac{1}{r^{2} \alpha} \frac{\partial^{2}(\cdot)}{\partial \varphi^{2}} .
$$

In addition, the motion is completely described by two coupled dynamical equations for $u_{B}$ and $\omega_{B}$ :

$$
\left\{\begin{aligned}
\partial_{t} u_{B}+N L_{u} & =V T_{u} \\
\partial_{t} \omega_{B}+N L_{\omega} & =V T_{\omega}
\end{aligned}\right.
$$

where $\partial_{t}$ stands for the time derivative. In the above equations, the nonlinear and viscous terms are expressed as

$$
\begin{gathered}
N L_{u} \equiv(\boldsymbol{\omega} \times \boldsymbol{u}) \cdot \boldsymbol{e}_{B}, \quad N L_{\omega} \equiv[\nabla \times(\boldsymbol{\omega} \times \boldsymbol{u})] \cdot \boldsymbol{e}_{B}, \quad V T_{u} \equiv v\left[\mathbb{L}\left(\frac{u_{B}}{\alpha}\right)-\frac{2 \alpha^{2}}{L} \omega_{B}\right] \\
V T_{\omega} \equiv-v[\nabla \times(\boldsymbol{\nabla} \times \boldsymbol{\omega})] \cdot \boldsymbol{e}_{B}=v\left[\mathbb{L}\left(\frac{\omega_{B}}{\alpha}\right)-\left(\frac{2 \alpha^{2}}{L}\right)^{2} \omega_{B}+\frac{2 \alpha^{2}}{L} \mathbb{L}\left(\frac{u_{B}}{\alpha}\right)\right]
\end{gathered}
$$

where $v$ stands for the kinematic viscosity of the fluid. Contrary to what occurs in the two-dimensional framework, the viscous terms $V T_{u}$ and $V T_{\omega}$ here couple the components $u_{B}$ and $\omega_{B}$. It is also convenient to introduce the quantity $u_{H}$ defined by

$$
u_{H} \equiv \frac{u_{B}}{\alpha}-\left(U_{z}^{\infty}+\frac{\Gamma}{2 \pi L}\right)
$$

where $U_{z}^{\infty}$ denotes the axial velocity far from the $z$-axis and $\Gamma$ the total flow circulation. Indeed, this latter quantity vanishes far from the vorticity region since $u_{B} / \alpha=\left(u_{z}+r u_{\theta} / L\right)$ behaves as $U_{z}^{\infty}+\Gamma /(2 \pi L)$ when $r \rightarrow \infty$. When $u_{H}$ is uniform in space, it is bound by definition to vanish everywhere.

A numerical code has been written to implement the time advance of equations (8) based on a $\Psi-\omega$ formulation generalized to the helical symmetry framework. This code is briefly outlined below but extensive details can be found in Ref. [26]. Quantities are represented in variables $r$ and $\varphi$. The code uses Fourier series along the $\varphi$ direction where a $2 \pi$-periodicity holds, and second order finite differences in the radial direction. The time advance is performed on each "azimuthal" mode using a second-order backward discretisation of the temporal derivative. Viscous terms are treated implicitly. Nonlinear terms appear explicitly through a second order Adams-Bashforth extrapolation. They are evaluated in the physical space, which involves the inverse Fourier transform of velocity and vorticity components, a standard 2/3 desaliasing procedure and a direct Fourier transform of nonlinear terms. The numerical domain has a radial extension of approximately 3 helix radii: the outer boundary is located at radial distance $R_{\text {ext }}=3$ made dimensionless with quantity $R_{\star}$ as stated below in $\S \mathrm{IV} \mathrm{A}$. The domain is meshed by $N_{r} \times N_{\theta}=500 \times 384$ grid points. The condition $U_{z}^{\infty}=0$ is imposed: this selects a specific reference frame which might differ from the laboratory frame in experiments. 
B. The helical solutions in the $\Pi_{\perp}$ plane

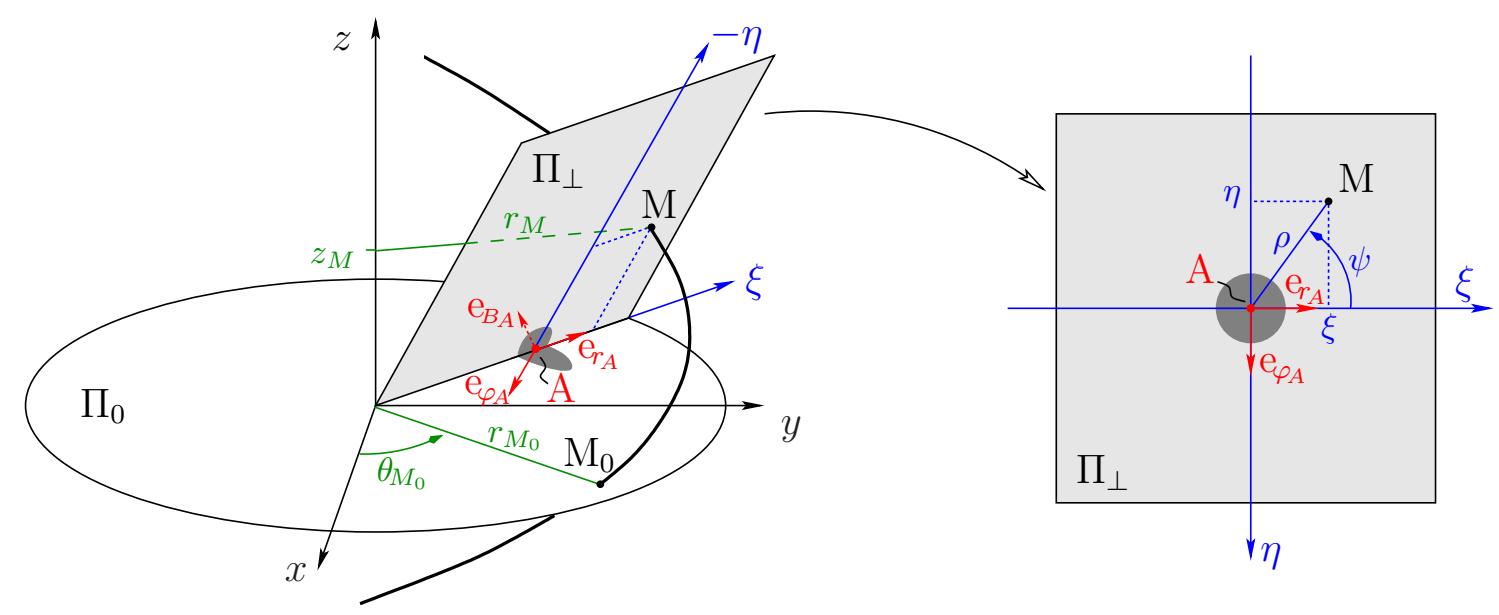

FIG. 2: Definition of planes $\Pi_{0}$ and $\Pi_{\perp}$, as well as of local bases and coordinates used for vortex characterization. The dark spots represent the vortex core cut by either plane. Note that the sketch is done for negative values of $\eta$ and $\psi$.

In order to characterize a helically symmetric flow solution, one may cut the system by any $(r, \theta)$ plane perpendicular to the $z$-axis, or by any meridional $(r, z)$ plane. Indeed, quantities such as helical velocity and vorticity components numerically obtained from the DNS at discrete points $\left(r_{i}, \varphi_{j}\right)$, may equally be viewed at points $\left(r_{i}, \theta_{j}=\varphi_{j}\right)$ in the plane $z=0$ (hereafter called $\left.\Pi_{0}\right)$ or at points $\left(r_{i}, z_{j}=-L \varphi_{j}\right)$ in the plane $\theta=0$ (hereafter called $\Pi_{z}$ ). Yet, neither $\Pi_{0}$ nor $\Pi_{z}$ are convenient cut planes in the helical context because the characterization of vortex cores there may be affected by a geometrical bias. For instance, a vortex possessing an axisymmetric core structure yields a non-circular pattern if the plane intercepts the vortex axis in a non-orthogonal fashion. In order to alleviate this problem, a more appropriate plane called $\Pi_{\perp}$ is introduced, which cuts the vortex "perpendicularly" at some point A where the helical vorticity component reaches its maximum, situated at distance $r_{A}(t)$ from the origin. At a given time $t$, plane $\Pi_{\perp}$ is then defined as the plane containing point $A$ and normal to the helical line passing through $A$, i.e. normal to the vector $\boldsymbol{e}_{B_{A}} \equiv \boldsymbol{e}_{B}\left(r_{A}, \theta_{A}\right)$ (figure 2 ). Any point $\mathrm{M}$ in the $\Pi_{\perp}$ plane is defined by Cartesian coordinates $(\xi, \eta)$ :

$$
\mathbf{A M}=\xi \boldsymbol{e}_{r_{A}}+\eta \boldsymbol{e}_{\varphi_{A}}
$$

where $\boldsymbol{e}_{r_{A}}$ and $\boldsymbol{e}_{\varphi_{A}}$ are the two other vectors of the Serret-Frénet basis (2) at point A. One can also use polar coordinates $(\rho, \psi)$ centered on $\mathrm{A}$, such that $\xi=\rho \cos \psi, \eta=\rho \sin \psi$ and the local polar basis $\left(\boldsymbol{e}_{\rho}, \boldsymbol{e}_{\psi}\right)$ is given by $\boldsymbol{e}_{\rho}=\cos \psi \boldsymbol{e}_{r_{A}}+\sin \psi \boldsymbol{e}_{\varphi_{A}}$ and $\boldsymbol{e}_{\psi}=-\sin \psi \boldsymbol{e}_{r_{A}}+\cos \psi \boldsymbol{e}_{\varphi_{A}}$.

In order to characterize the vortex in the $\Pi_{\perp}$ plane, fields are determined in this plane using a discrete polar grid: $\rho_{p}$ is evenly spaced $\left(p=0 \cdots N_{\rho}-1\right)$ between 0 and a few typical vortex core sizes, and $\psi_{q}=2 \pi q / N_{\psi}$ $\left(q=0 \cdots N_{\psi}-1\right)$. To do so, the numerical data obtained on the $\left(r_{i}, \varphi_{j}\right)$ grid is transfered to the $\left(\rho_{p}, \psi_{q}\right)$ grid in the $\Pi_{\perp}$ plane. This involves geometrical transforms and accurate interpolations which are explained in detail in appendix A. Figures $3 a$ and $b$ display examples of vortex cores in the plane $\Pi_{\perp}$ : the vortex 
(a)

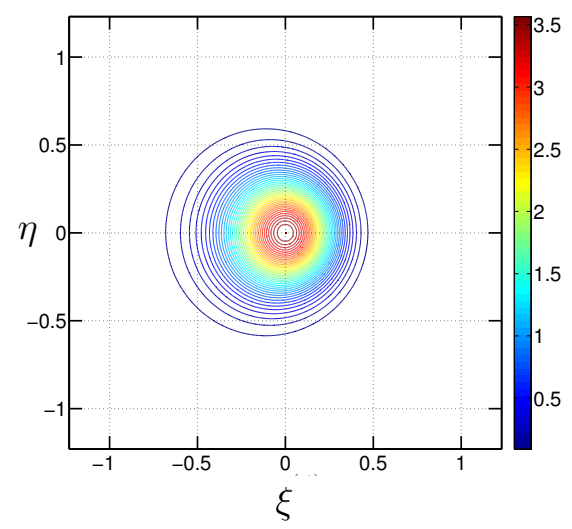

(b)

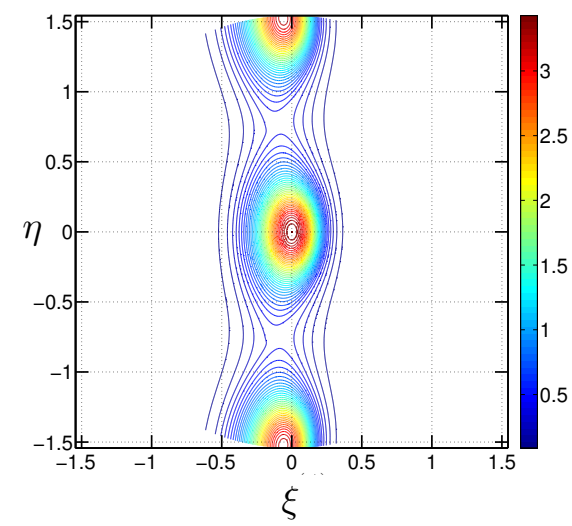

FIG. 3: Helical vortex of reduced pitch (a) $L=1$ and (b) $L=0.25$ : isocontours of $\omega_{B_{A}}$ in the plane $\Pi_{\perp}$. Both vortices have comparable core sizes.

core is generally not purely axisymmetric and this dynamical feature due to vortex curvature becomes more significant at low $L$.

In the plane $\Pi_{\perp}$, the vorticity field is separated into a component orthogonal to the plane $\Pi_{\perp}$, namely $\omega_{B_{A}} \equiv \boldsymbol{\omega} \cdot \boldsymbol{e}_{B_{A}}$ (except at point A, $\omega_{B_{A}}$ is different from $\omega_{B}$ ), and two in-plane polar components $\omega_{\rho} \equiv \boldsymbol{\omega} \cdot \boldsymbol{e}_{\rho}$ and $\omega_{\psi} \equiv \boldsymbol{\omega} \cdot \boldsymbol{e}_{\psi}$. The core structure is studied by decomposing these vorticity components in multipolar contributions i.e. using a discrete Fourier transform in the azimuthal direction $\psi$. For instance, quantity $\omega_{B_{A}}\left(\rho_{p}, \psi_{q}\right)$ is decomposed in azimuthal modes of wavenumber $m$ :

$$
\omega_{B_{A}}^{(m)}\left(\rho_{p}\right)=\frac{1}{N_{\psi}} \sum_{q=0}^{N_{\psi}-1} \omega_{B_{A}}\left(\rho_{p}, \psi_{q}\right) \mathrm{e}^{-\mathrm{i} m \psi_{q}}
$$

Such decomposition has been successfully applied to characterize the instantaneous deformation of helical vortices by comparison with the theoretical results of an asymptotic study [25]. Here, it is applied to characterize vorticity $\omega_{B_{A}}^{(0)}(r, t)$ as well as velocity $u_{H}{ }^{(0)}(r, t)$ profiles, core radius and ellipticity (see discussion in appendix B 2 and results in section $\mathrm{V})$.

\section{CONSERVATION PROPERTIES FOR VISCOUS OR INVISCID HELICAL FLOWS}

In this section, some invariant quantities and conservation properties are derived from the inviscid or viscous dynamics of helical flows. 


\section{A. Global quantities for viscous flow: some exact relations}

Global quantities are obtained by integration in any $\Pi_{0}$ plane over a disk $S$ having a radius $R_{\text {ext }}$ large enough to encompass the region of nonzero vorticity. Quantities $u_{H}, \omega_{B}$ and $\omega_{z}$ are assumed to tend to zero rapidly enough as $r \rightarrow \infty$, so that the global quantities presented hereafter are convergent integrals, i.e. independent of the selected value of $R_{\text {ext }}$. Here we introduce two exact global viscous invariants and a global quantity which evolves linearly in time.

The total circulation

$$
\Gamma=\iint_{S} \omega_{z} r \mathrm{~d} r \mathrm{~d} \theta
$$

is known to be a global viscous invariant. Let us insert the equality for $\omega_{z}$ valid in the helical symmetry context

$$
\omega_{z}=\alpha\left(\omega_{B}-\frac{r}{L} \omega_{\varphi}\right) \text { with } \omega_{\varphi}=-\alpha \frac{\partial u_{H}}{\partial r}
$$

into equation (14). After an integration by parts, one is led to

$$
\Gamma=\mathscr{K}_{1}-\frac{2}{L} \mathscr{K}_{2} \quad \text { where } \quad \mathscr{K}_{1} \equiv \iint_{S} \alpha \omega_{B} r \mathrm{~d} r \mathrm{~d} \theta, \quad \mathscr{K}_{2} \equiv \iint_{S} \alpha^{4} u_{H} r \mathrm{~d} r \mathrm{~d} \theta .
$$

The axial momentum $\mathscr{P}_{z}$ per axial length unit

$$
\mathscr{P}_{z}=\iint_{S} r \omega_{\theta} r \mathrm{~d} r \mathrm{~d} \theta \quad \text { with } \omega_{\theta}=\alpha\left(\omega_{\varphi}+\frac{r}{L} \omega_{B}\right) .
$$

is another global viscous invariant $[29,30]$. In the context of helical symmetry, this invariant reads:

$$
\mathscr{P}_{z}=2 \mathscr{K}_{2}+\frac{1}{L} \mathscr{K}_{3}, \text { where } \mathscr{K}_{3} \equiv \iint_{S} r^{2} \alpha \omega_{B} r \mathrm{~d} r \mathrm{~d} \theta
$$

Upon eliminating $\mathscr{K}_{2}$ from (16) and (18), an integral equation for $\omega_{B}$ only is found:

$$
\iint_{S} \frac{\omega_{B}}{\alpha} r \mathrm{~d} r \mathrm{~d} \theta=\Gamma+\frac{\mathscr{P}_{z}}{L}=\text { const. }
$$

A third global quantity is also of interest: the global angular momentum $\mathscr{L}_{z}$ per axial length unit

$$
\mathscr{L}_{z} \equiv \iint_{S} r^{2} \omega_{z} r \mathrm{~d} r \mathrm{~d} \theta
$$

In the context of helical symmetry, $\mathscr{L}_{z}$ can be written in terms of integrals of $\omega_{B}$ and $u_{H}$ as:

$$
\mathscr{L}_{z}=2 L \mathscr{K}_{2}+\mathscr{K}_{3}-2 L \mathscr{K}_{4}, \text { where } \mathscr{K}_{4} \equiv \iint_{S} u_{H} r \mathrm{~d} r \mathrm{~d} \theta .
$$

It can be shown from the Navier-Stokes equations that this quantity is not an invariant but evolves according to

$$
\mathscr{L}_{z}(t)=\mathscr{L}_{z}(0)+4 \Gamma v t
$$


Relations (18), (21) and (22) can be combined to yield the following time evolution for the integral of $u_{H}$ :

$$
\iint_{S} u_{H} r \mathrm{~d} r \mathrm{~d} \theta=\underbrace{\frac{1}{2}\left[\mathscr{P}_{z}-\frac{\mathscr{L}_{z}(0)}{L}\right]}_{\text {const. }}-\frac{2 \Gamma}{L} v t .
$$

For flows with zero total circulation $(\Gamma=0)$, the global angular momentum $\mathscr{L}_{z}$ is conserved [29], in which case, due to property (23), the integral $\mathscr{K}_{4}$ of $u_{H}$ becomes time-independent.

\section{B. Exact local conservation laws in the inviscid framework}

Apart from global quantities, it is worth mentioning two exact local conservation laws for helically symmetric flows. For inviscid flows [24], quantity $u_{H}$ is materially conserved [see equations (8) and (9)]:

$$
\partial_{t} u_{H}+u_{r} \frac{\partial u_{H}}{\partial r}+\frac{u_{\varphi}}{\alpha r} \frac{\partial u_{H}}{\partial \varphi}=0
$$

Using (4), the above equation may be re-written as

$$
\partial_{t} u_{H}+J\left(u_{H}, \Psi\right)=0, \quad \text { where } J(f, g) \equiv \frac{1}{r}\left[\frac{\partial f}{\partial r} \frac{\partial g}{\partial \varphi}-\frac{\partial f}{\partial \varphi} \frac{\partial g}{\partial r}\right] .
$$

This implies that a helical flow with $u_{H}=0$ everywhere remains so in time if $v=0$. In such flows, vorticity remains everywhere tangent to helical lines since vorticity components $\omega_{r}$ and $\omega_{\varphi}$ are uniformly zero (see equations (5) and (11)).

In the inviscid framework, it was further showed [24] that quantity $\alpha \omega_{B}$ evolves according to

$$
\partial_{t}\left(\alpha \omega_{B}\right)+J\left(\alpha \omega_{B}, \Psi\right)+\frac{2 \alpha^{4}}{L} J\left(u_{H}, \Psi\right)+\frac{2 \alpha^{4}}{L^{2}} u_{H} \frac{\partial u_{H}}{\partial \varphi}=0 .
$$

Contrary to what happens for $\omega_{z}$ in the two-dimensional case (obtained in the limit $L=\infty$ ), quantity $\alpha \omega_{B}$ is not conserved on a general basis. However, when $u_{H}$ is uniformly zero, $\alpha \omega_{B}$ is materially conserved:

$$
\partial_{t}\left(\alpha \omega_{B}\right)+J\left(\alpha \omega_{B}, \Psi\right)=0 \text { when } u_{H}=0 .
$$

Similarly to the case of vortex rings, this equation possesses a geometrical interpretation in terms of an infinitesimal helical vortex tube of radius $r(t)$ and transversal section $\delta A(t)$. When $u_{H}=0$, volume conservation of one helix turn imposes that $\delta A(t) \sqrt{r^{2}+L^{2}}$ is constant, and circulation invariance imposes that $\omega_{B} \delta A(t)$ is constant as well. Both relations lead to the material conservation of $\alpha \omega_{B}$ if $u_{H}=0$.

\section{Approximate local conservation laws in the viscous framework}

When viscosity is present, the equations for $u_{H}$ and $\alpha \omega_{B}$ respectively read

$$
\partial_{t} u_{H}+J\left(u_{H}, \Psi\right)=\frac{v}{\alpha} \mathbb{L}\left(u_{H}\right)-\frac{2 v}{L} \alpha \omega_{B}
$$


and

$$
\begin{array}{r}
\partial_{t}\left(\alpha \omega_{B}\right)+J\left(\alpha \omega_{B}, \Psi\right)+\frac{2 \alpha^{4}}{L} J\left(u_{H}, \Psi\right)+\frac{2 \alpha^{4}}{L^{2}} u_{H} \frac{\partial u_{H}}{\partial \varphi} \\
\quad=v\left[\frac{1}{\alpha} \mathbb{L}\left(\alpha \omega_{B}\right)+\frac{4 r \alpha^{2}}{L^{2}} \frac{\partial}{\partial r}\left(\alpha \omega_{B}\right)+\frac{2 \alpha^{3}}{L} \mathbb{L}\left(u_{H}\right)\right] .
\end{array}
$$

The last term on the r.h.s. of equation (28) implies that, contrary to the inviscid case, $u_{H}$ cannot remain zero if $\left.u_{H}\right|_{t=0}=0$, in presence of helical vorticity. It is possible, however, to find an approximate time evolution in the viscous case. Indeed, when $\left.u_{H}\right|_{t=0}=0$, equation (23) imposes $\mathscr{P}_{z}=\mathscr{L}_{z} / L$ and thus suggests that $u_{H}$ might depend linearly on $v t$. Following this idea, we neglect for the initial time period the $u_{H}$ terms in the dynamic equation (29) for $\alpha \omega_{B}$, leading to

$$
\partial_{t}\left(\alpha \omega_{B}\right)+J\left(\alpha \omega_{B}, \Psi\right) \approx v\left[\frac{1}{\alpha} \mathbb{L}\left(\alpha \omega_{B}\right)+\frac{4 r \alpha^{2}}{L^{2}} \frac{\partial}{\partial r}\left(\alpha \omega_{B}\right)\right] .
$$

If $\omega_{B}$ is associated to a vortex of core size $a \ll R_{\star}$, it is possible to evaluate the order of magnitude of the two terms in the r.h.s. of (30):

- for $L \ll R_{\star}, \alpha$ is of order $L / R_{\star}$ and

$$
\frac{v}{\alpha} \mathbb{L}\left(\alpha \omega_{B}\right) \sim v \omega_{B} \frac{L}{R_{\star} a^{2}} \text { and } \frac{4 v r \alpha^{2}}{L^{2}} \frac{\partial}{\partial r}\left(\alpha \omega_{B}\right) \sim v \omega_{B} \frac{L}{R_{\star}^{2} a} .
$$

The second term is negligible with respect to the first one since $a \ll R_{\star}$.

- for $L \gg R_{\star}$ or $L=O\left(R_{\star}\right), \alpha$ is of order 1 and thus

$$
\frac{v}{\alpha} \mathbb{L}\left(\alpha \omega_{B}\right) \sim v \omega_{B} \frac{1}{a^{2}} \text { and } \frac{4 v r \alpha^{2}}{L^{2}} \frac{\partial}{\partial r}\left(\alpha \omega_{B}\right) \sim v \omega_{B} \frac{R_{\star}}{L^{2} a} .
$$

The second term can be neglected with respect to the first one if $a \ll L^{2} / R_{\star}$. This always holds for $L \gg R_{\star}$ or $L=O\left(R_{\star}\right)$ since again $a \ll R_{\star}$.

When $a \ll R_{\star}$, the first term in the r.h.s. of (30) is thus dominant with respect to the second one, and $\alpha \omega_{B}$ satisfies

$$
\partial_{t}\left(\alpha \omega_{B}\right)+J\left(\alpha \omega_{B}, \Psi\right) \approx \frac{v}{\alpha} \mathbb{L}\left(\alpha \omega_{B}\right) .
$$

It can be easily checked that the exact equation (28) and approximate equation (33) together with the initial condition $\left.u_{H}\right|_{t=0}=0$ are simultaneously satisfied if the following relationship holds between $\alpha \omega_{B}(r, \varphi, t)$ and $u_{H}(r, \varphi, t)$ :

$$
u_{H}=-\frac{2 v t}{L} \alpha \omega_{B} .
$$

Relation (34) is thus approximate and valid whenever $u_{H}$ remains small. Note that it is also consistent with the exact integral relation (23). This idea can be extended to a solution in which the initial condition reads 
as

$$
\left.u_{H}\right|_{t=0}=-\left.\frac{\delta^{2}}{2 L} \alpha \omega_{B}\right|_{t=0},
$$

where $\delta$ is a length such that $\delta / L \ll 1$. In that case, the same approximation leads to the solution

$$
u_{H}(r, \varphi, t)=-\frac{\delta^{2}+4 v t}{2 L} \alpha \omega_{B}(r, \varphi, t) .
$$

This ansatz is indeed observed in the numerical results of $\S \mathrm{VC}$.

\section{HELICAL VORTEX: REACHING QUASI-EQUILIBRIUM}

\section{A. Initial conditions}

For a two-dimensional vortex, a known solution of the Navier-Stokes equations is

$$
\omega_{z}=\frac{\Gamma}{\pi a^{2}} \exp \left[-\rho^{2} / a^{2}(t)\right], \quad \text { with } a(t)=\left(a_{0}^{2}+4 v t\right)^{1 / 2} .
$$

This solution can be seen as the viscous speading of a vortex generated by a Dirac singularity at time $t_{\star}=-\frac{1}{4} a_{0}^{2} / v<0$. Introducing the shifted time $\tau \equiv t-t_{\star}$ makes the diffusion law independent on the initial core size $a_{0}$. Let us now extend this idea to obtain a single helical vortex solution generated from a singular helical filament. This singular filament is characterized by its circulation $\Gamma$, its reduced pitch $L$ and the helix radius $R_{\star}$. Contrary to the two-dimensional case, no analytical viscous solution is available corresponding to the viscous spreading of a singular helical vortex. Nevertheless conservation properties derived in section III are useful to elaborate the specific initial conditions that may lead to generic quasi-equilibrium states. In particular, such solutions should preserve circulation $\Gamma$ and axial momentum $\mathscr{P}_{z}$.

At short times, vorticity is concentrated around point $\mathrm{A}$ in a region of the plane $\Pi_{\perp}$ the extent of which is much smaller than $L$ and $R_{\star}$. It is thus reasonable to assume that the two-dimensional diffusion law is satisfied in the $\Pi_{\perp}$ plane. This implies that $(i)$ the initial vorticity profile in the $\Pi_{\perp}$ plane may be assumed to be Gaussian:

$$
\left.\omega_{B}\right|_{t=0}=C_{0} \exp \left[-\left(\rho / a_{0}\right)^{2}\right] ;
$$

(ii) this solution is the outcome of a helical singular vortex at time $t_{\star}=-\frac{1}{4} a_{0}^{2} / v$. This also implies that the velocity component $u_{H}$ is linked to $\omega_{B}$ by the ansatz (35) with $\delta=a_{0}$, namely:

$$
\left.u_{H}\right|_{t=0}=-\left.\frac{a_{0}^{2}}{2 L} \alpha \omega_{B}\right|_{t=0} .
$$

At this stage, the normalisation constant $C_{0}$ in (37) is yet to be determined. This is also the case for the helix radius $r_{A}(t=0)$ since $r_{A}$ is unsteady and a priori different from $R_{\star}$. In order to determine both quantities, one uses the following properties: (i) the prescribed circulation $\Gamma$ and axial momentum $\mathscr{P}_{z}$ are conserved during the time evolution, (ii) when $t \rightarrow t_{\star}$, i.e. in the limit of the singular filament, $r_{A}(t) \rightarrow R_{\star}$ and $\mathscr{P}_{z}=R_{\star}^{2} \Gamma / L$. Using both remarks, $C_{0}$ and $r_{A}(t=0)$ can be computed: guess values are assumed, then $C_{0}$ and $r_{A}(t=0)$ are iteratively adjusted until circulation and axial momentum evaluated through formulas (16) and (18) converge to their prescribed values $\Gamma$ and $R_{\star}^{2} \Gamma / L$. 
From now on, variables are made nondimensional using $R_{\star}$ as a lengthscale, $\Gamma / R_{\star}$ as a timescale. Simulations a priori depend on three dimensionless parameters: the Reynolds number $\Gamma / v$, the dimensionless reduced pitch $L$ and the dimensionless core size $a_{0}$. We will show in the subsequent subsection that the dependence on $a_{0}$ can indeed be removed by using the time shift $\tau=t-t_{\star}$ as postulated above.

\section{B. Transient evolution}

The present paper focuses on the time evolution of a single helical vortex starting from the specific initial conditions (37)-(38). This initial profile, purely axisymmetric in the $\Pi_{\perp}$ plane, is a priori not an Euler equilibrium. Such situation is reminiscent of the evolution of an initial polygonal array of identical, well separated two-dimensional Gaussian vortices. In such flows, apart from the solid-like rotation, each vortex evolves due to diffusion, but also due to the presence of an external strain arising from mutual interactions. This occurs in two stages, as observed for co-rotative [31] and counter-rotating [32] vortices: a first rapid relaxation in which damped Kelvin waves are observed to propagate on the vortices, and a second phase in which a quasi-equilibrium is reached that slowly diffuses. In this latter stage, it is found that the time evolution of each vortex does not depend on $a_{0}$ if expressed as a function of $\tau$.

Similarly to what happens for such a vortex array, a helical vortex is subjected to rotation and strain, though it is now self-induced. Based on this analogy, the present section shows that this two-step process also prevails for a single helical vortex.

The rapid relaxation is presented for a helical vortex of pitch $L=0.25$, of initial core size $a_{0}=0.06$, at $R e=5000$. The evolution is similar at other pitch values and Reynolds numbers. The time evolution of the helical vortex is displayed in figure 4 where the vorticity component $\omega_{B_{A}}$ is presented in the $\Pi_{\perp}$ plane. Snapshots show how the initial axisymmetric vorticity distribution becomes asymmetric within the core, while the very weak peripheral vorticity region displays a complex evolution associated to the damping of inertial waves by viscosity. The vortex adapts its structure to the self-induced strain field originating from local curvature as well as induction due to remote vorticity.

The above process leads to a quasi-equilibrium. The genericity of this state with respect to the initial condition is illustrated in figure $5 \mathrm{a}$ and $\mathrm{b}$. Results are identical when computations for pitches $L=0.25$ and $L=1$ are started with two different initial core sizes $a_{0}=0.05,0.1$ (corresponding to the values $\tau=-t_{\star}=3.12,12.5$ ). In figure 5a, the time evolution of $r_{A}$ is plotted as a function of the shifted time $\tau$. For a given $L$, all the curves end up collapsing after a short transient period (due to Kelvin waves and characterized by the wiggles). Note that, even if the initial oscillations tend to increase in amplitude and duration as the initial core size is increased or the pitch $L$ is decreased, they remain limited. Moreover, one may estimate the vortex core size at each time of the process. How this quantity is computed is precisely explained in appendix $\mathrm{B}$. The core size in figure $5 \mathrm{~b}$ follows the same curve for two initial core sizes. The curve is very close to the two-dimensional diffusion law for short times $\tau$, and then departs from it (for this latter point, see $\S \mathrm{VA}$ ). A contrario, when $a_{0}$ is too large (figure $5 \mathrm{c}$ ), there is a slight shift with respect to the curves emanating from the small initial core sizes, which could be accounted for by correcting the evaluation of $t_{\star}$. This point is even more underlined when $L$ is small and self-interactions become stronger.

The point vortex diffusion hypothesis coupled to the ansatz (35) for $u_{H}$ thus leads to a generic quasiequilibrium state: the long-time dynamics do not depend on the initial core size $a_{0}$ when small. In the following, we use the initial core size $a_{0}=0.1$. The number of control parameters is thus reduced to only two: the reduced pitch $L$ and the Reynolds number $R e$. 

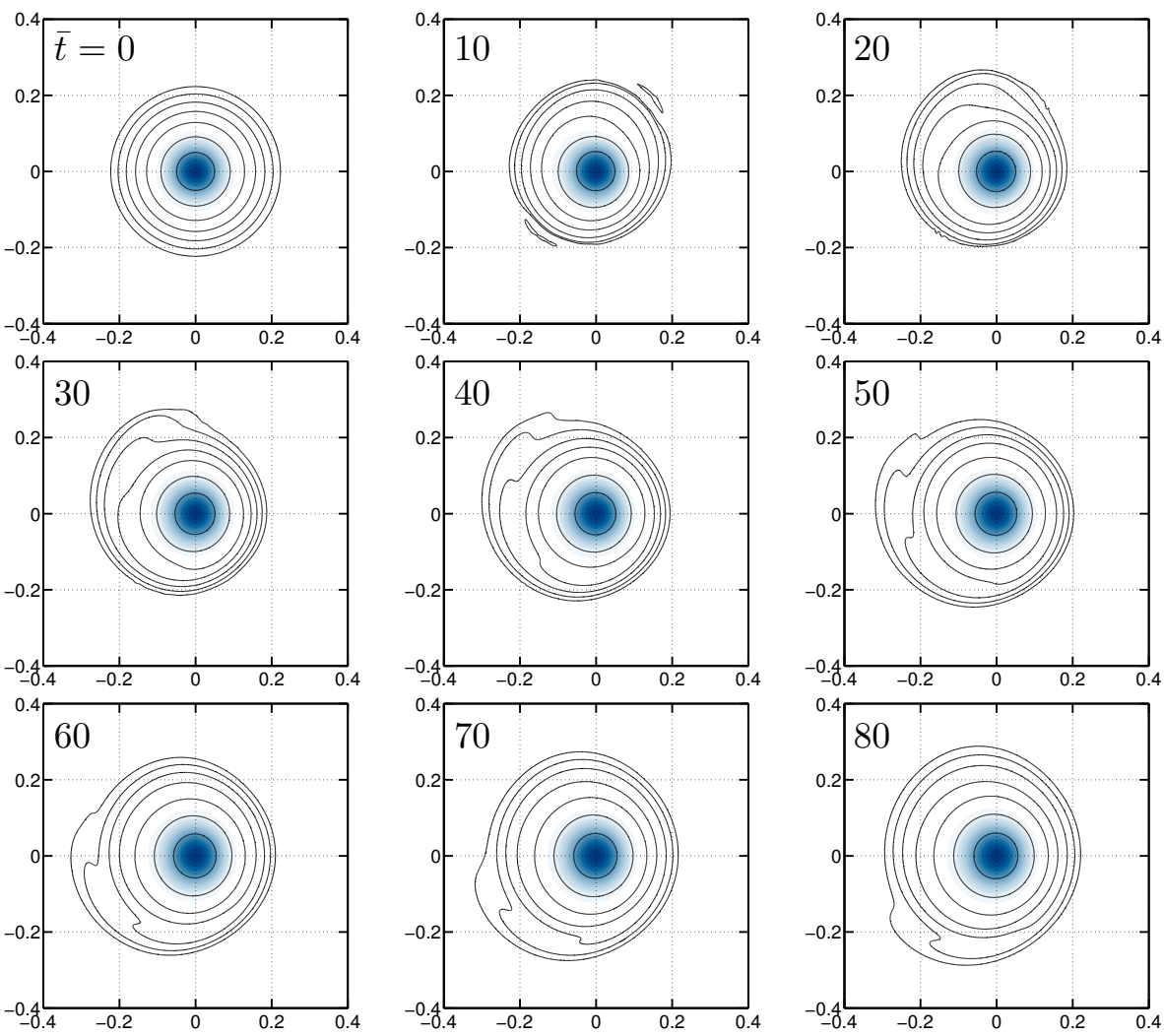

FIG. 4: Helical vortex of pitch $L=0.25$ at $R e=5000$ for $a_{0}=0.06$. Vorticity contours in the $\Pi_{\perp}$ plane during the relaxation process, for times $\bar{t} \equiv t \Gamma /\left(2 \pi a_{0}^{2}\right)=0,10, \cdots, 80$. Contour levels are

$$
\omega_{B_{A}} / \omega_{B_{A}}^{(0)}(0)=0.5,10^{-1}, 10^{-2}, \cdots, 10^{-6} .
$$

\section{QUASI-EQUILIBRIUM OF A HELICAL VORTEX}

The lack of explicit solutions of the helical Navier-Stokes equations justifies that helical vortex quasiequilibria computed by DNS need to be accurately described. As the geometry of such flows is rather involved, specific characterization techniques are employed to determine helix radius, angular velocity. In addition to core radius, ellipticity can be also computed since vortex core are not purely axisymmetric (see figures $3 b$ and d). All these quantities are defined in the appendix B as well as the numerical procedures used to compute them. In the present section, generic quasi-equilibrium states obtained for various pitches between $L=0.25$ and $L=3$ at fixed Reynolds number $R e=5000$ are characterized. 
(a)

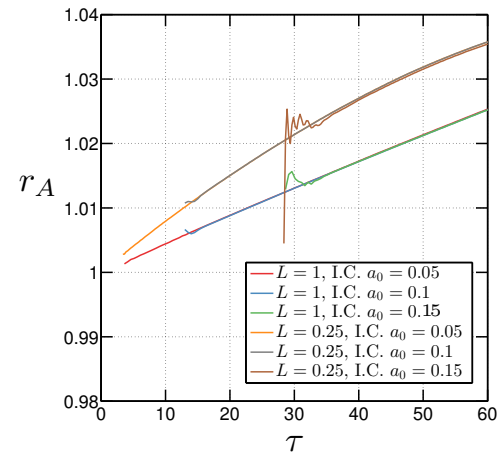

(b)

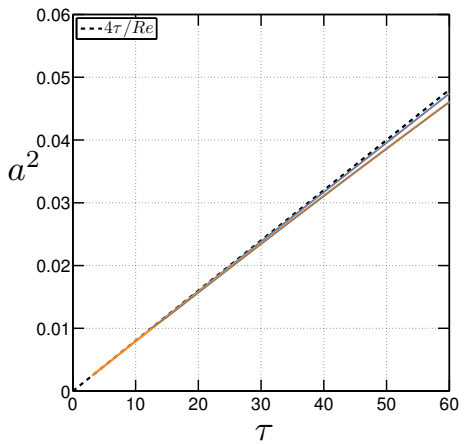

(c)

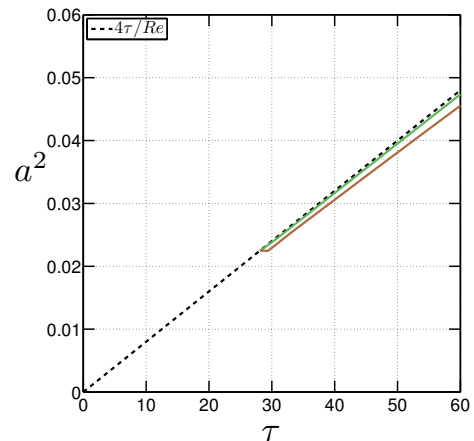

FIG. 5: Helical vortex of pitch $L=1$ and $L=0.25$ at $R e=5000$. (a) Time evolution of the radial position $r_{A}$ of the maximum of vorticity $\omega_{B}$ as a function of $\tau$, for three different core sizes $a_{0}=0.05,0.1,0.15$ corresponding to initial times $\tau=3.125,12.5$ and 28.125. (b) Time evolution of the squared core size $a^{2}(\tau)$ for the two core sizes $a_{0}=0.05$ and 0.1 (the black dashed line is the 2D diffusion law $4 \tau / \operatorname{Re}$ ). (c) Same as (b), but for the largest initial core size $a_{0}=0.15$.

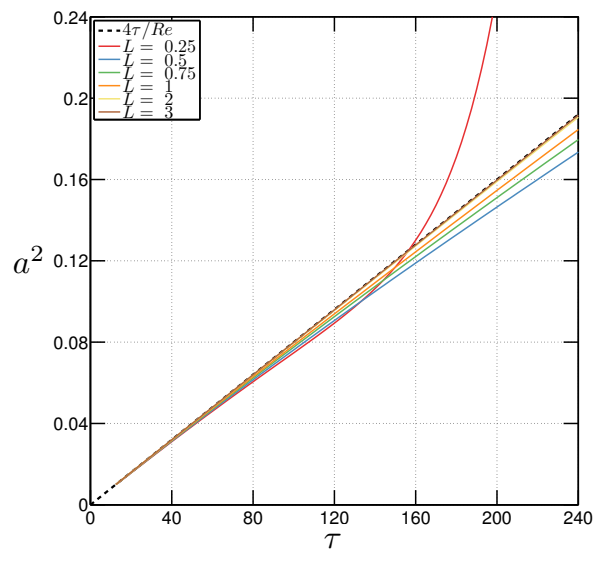

FIG. 6: Helical vortex for different values of $L$ between 0.25 and 3. Time evolution of the squared core size $a^{2}$ at $R e=5000$. The black dashed line shows the 2D diffusion law $4 \tau / \operatorname{Re}$.

\section{A. Core radius \& self-similarity}

The core size $a$ of a helical vortex is computed using the technique based on the fit of the axisymmetric part of the helical vorticity in plane $\Pi_{\perp}$ (for details see appendix B 2 ). When $L>1$, the two-dimensional diffusion law is a fair approximation for the core size evolution, as can be seen in figure 6 . When $L<1$, the 
core size increases less than its two-dimensional counterpart. The strong increase of $a$ observed for $L=0.25$, around $\tau=130$, corresponds to situations where coils significantly overlap $(a \geq 0.32>L)$ making the notion of individual core size inadequate.

(a)

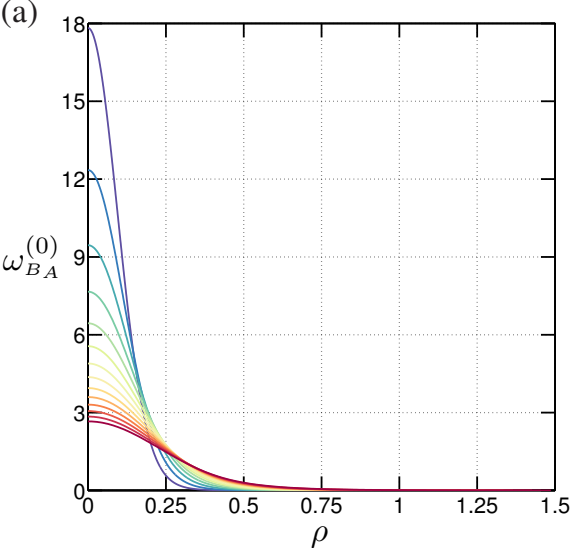

(b)

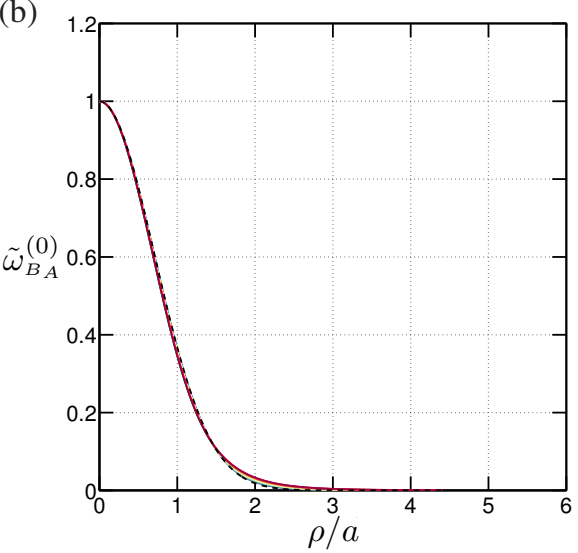

FIG. 7: Helical vortex of pitch $L=0.5$ at $R e=5000$. (a) Axisymmetric part of the helical vorticity $\omega_{B_{A}}^{(0)}(\rho)$ at times $\tau=22.5,32.5, \ldots, 172.5$ (amplitude decreases with time). (b) Same profiles normalized in amplitude by the maximum value at each time, as a function of the similarity variable $\bar{\rho}=\rho / a$. Black dashed line: $\tilde{\omega}_{B_{A}}^{(0)}(\bar{\rho})=\mathrm{e}^{-\bar{\rho}^{2}}$.

Figure 7a displays the spreading of $\omega_{B_{A}}^{(0)}$ (i.e. the axisymmetric part of vorticity component $\boldsymbol{\omega} \cdot \boldsymbol{e}_{B_{A}}$ ) in time. When rescaled as follows:

$$
\tilde{\omega}_{B_{A}}^{(0)}=\frac{\omega_{B_{A}}^{(0)}(\bar{\rho}, \tau)}{\omega_{B_{A}}^{(0)}(0, \tau)}, \quad \bar{\rho}=\frac{\rho}{a(\tau)},
$$

these profiles collapse onto a Gaussian curve $\tilde{\omega}_{B_{A}}^{(0)}(\bar{\rho})=\exp \left(-\bar{\rho}^{2}\right)$ (figure 7b). This self-similarity has been predicted for viscous curved thin core vortices by Callegari \& Ting [33] and observed on numerically computed rotor wakes by Ali \& Abid [7].

Furthermore, the profiles of $u_{H}^{(0)}$ (i.e. the axisymmetric part of quantity $u_{H}$ ) spread in time with the same selfsimilar radial variable $\bar{\rho}$ (figure 8 ). Contrary to $\omega_{B_{A}}^{(0)}$, the amplitude of the velocity deficit $u_{H}^{(0)}(\rho=0, \tau)$ remains approximately constant in time (see value -0.14 for $L=0.5$ in figure $8 \mathrm{a}$ ). This can be accounted for using equation (34) coupled to the selfsimilarity (39) with $a^{2}(\tau) \approx 4 \tau / \operatorname{Re}$. The value $u_{H}^{(0)}(\rho=0)$ can be obtained with the following analytical argument: in the limit $a \rightarrow 0$, the radius $r_{A}$ tends to $1, \omega_{B}^{(0)}$ tends to $\exp \left[-(\rho / a)^{2}\right] /\left(\pi a^{2}\right)$, and, according to equation (34), $u_{H}^{(0)}$ tends to $-\alpha \exp \left[-(\rho / a)^{2}\right] /(2 \pi L)$. This implies that

$$
u_{H}^{(0)}(\rho=0) \rightarrow-\frac{1}{2 \pi \sqrt{1+L^{2}}}
$$


(a)

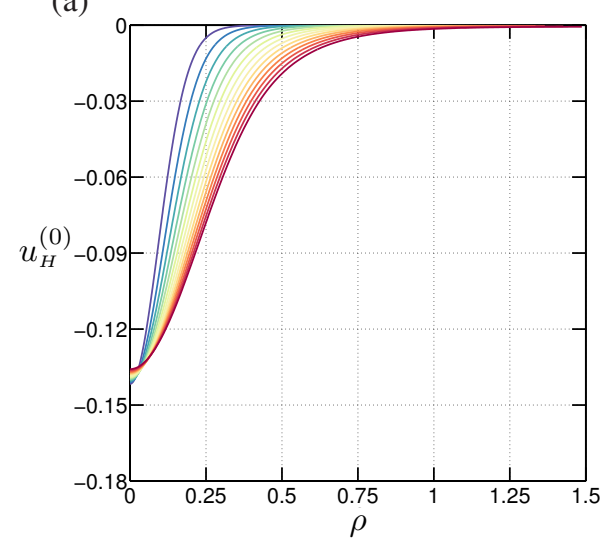

(b)

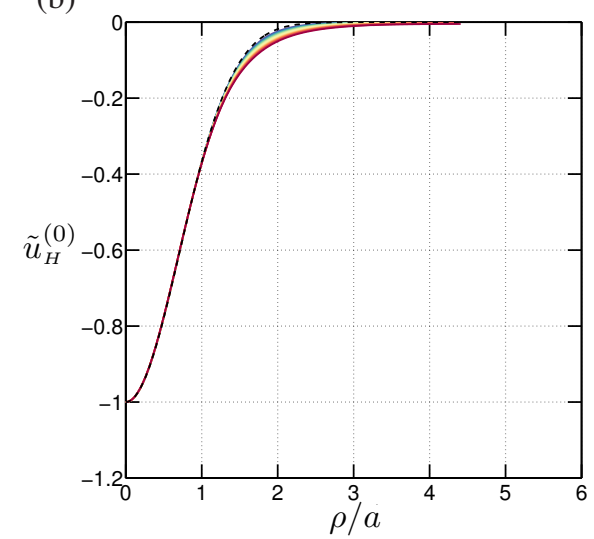

FIG. 8: Helical vortex of reduced pitch $L=0.5$ at $R e=5000$. (a) Axisymmetric part of the quantity $u_{H}(\rho)$ at times $\tau=22.5,32.5, \ldots, 172.5$ (the profile spreads as time increases). (b) Profiles rescaled by the maximum absolute value at each time, as a function of the similarity variable $\bar{\rho}=\rho / a$. Black dashed line:

$$
\tilde{u}_{H}^{(0)}=-\mathrm{e}^{-\bar{\rho}^{2}} .
$$

In the present case, $L=0.5$ yields $u_{H}(0) \approx-0.142$.

\section{B. Helix radius $r_{A}$ and angular velocity $\Omega$}

The temporal evolution of the helix radius $r_{A}$ is plotted in figure 9 for different values of $L$ at $R e=5000$. For all values of $L$ considered, the helix radius first increases, reaches a maximum and then decreases. During the period of increase, the rate $\mathrm{d} r_{A} / \mathrm{d} t$ is found to increase as $L$ is decreased, and seems to reach an asymptote value for small $L$. This value is found to be $7.1 \cdot 10^{-4}$, smaller than the theoretical value prevailing for a vortex ring $4.5902739 / R e \approx 9.2 \cdot 10^{-4}$ at asymptotically large $R e$ [34]. In vortex rings, this radial drift was related to the conservation of axial momentum [34]. For helical vortices, a similar argument based on the conservation of integral (17) together with the equation (22) for $\mathscr{L}_{z}(t)$ may hold, but its derivation probably necessitates to study the precise dipolar and quadrupolar structure of the fields, which is far beyond the scope of the paper.

For small $L$, the maximum is reached at a critical time where the vortex successive coils are about to overlap, as depicted in figure 10a and $\mathrm{b}$. For larger $L$, the same argument does not apply (see figure 10c and d): in this case, $r_{A}$ should tend to zero for large times since the asymptotic state is a columnar vortex centered on the $z$-axis. As a consequence, a maximum of $r_{A}$ is also expected.

The slow time evolution of angular velocity $\Omega$ is plotted in figure $9 \mathrm{c}$ and is compared to the value obtained with the cut-off theory [29]. This theory predicts a frequency $\Omega_{c}$ given by:

$$
\Omega_{c}(a, L)=-\frac{\Gamma}{2 \pi L^{2}}\left[\left(1+r_{A}^{2} / L^{2}\right) I\left(r_{A} / L, \delta / L\right)-K\left(r_{A} / L, \delta / L\right)\right],
$$


(a)

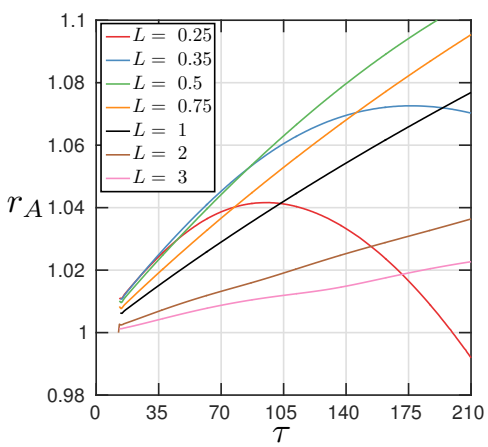

(b)

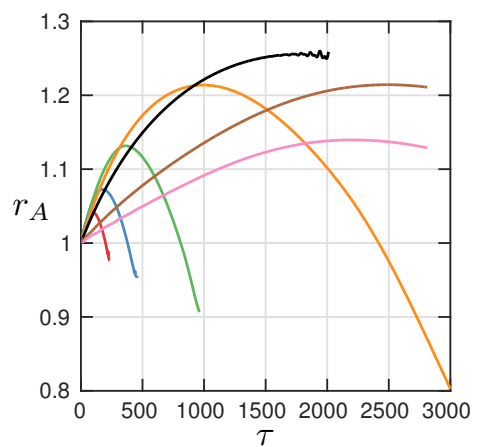

(c)

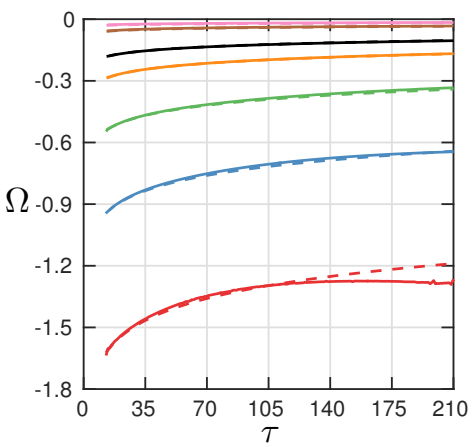

FIG. 9: Helical vortex for different values of $L$ between 0.25 and 3 , at $R e=5000$. (a) Helix radius $r_{A}$ as a function of $\tau$. (b) Same as (a) over a longer time period. (c) Angular velocity $\Omega$ as a function of $\tau$; the dashed lines are the values $\Omega_{c}$ predicted by the cut-off theory.

with

$$
\begin{aligned}
& K=\frac{L^{2}}{r_{A}^{2}}\left\{1-\frac{\theta_{0}}{\left[2 r_{A}^{2}\left[1-\cos \theta_{0}\right] / L^{2}+\theta_{0}^{2}\right]^{1 / 2}}\right\} \\
& I=\int_{\theta_{0}}^{\infty} \frac{[1-\cos \theta]}{\left\{\theta^{2}+2 r_{A}^{2}[1-\cos (\theta)] / L^{2}\right\}^{3 / 2}} \mathrm{~d} \theta .
\end{aligned}
$$

Here the terms $I$ and $K$ result from the self induced velocity due to the distant part of the vortex filament, $\theta_{0}$ is a cut-off angle defined using the core size $a$, the reduced pitch $L$ and a cut-off parameter for Gaussian vorticity distribution without axial flow $\delta=0.8735$ :

$$
\theta_{0}=\frac{a \delta}{|L| \sqrt{1+r_{A}^{2} / L^{2}}} .
$$

In (41)-(44), the value of $r_{A}$ is given by the DNS, and $a$ is approximated by a two-dimensional diffusion law. Figure $9 \mathrm{c}$ shows a good agreement for all values of $L$ except after the critical instant where $r_{A}$ reaches a maximum. This is expected since these dynamics cannot be captured by the filament model any longer and the two-dimensional diffusion law breaks down.

\section{Univoque relationship between $u_{H}$ or $\alpha \omega_{B}$ and $\Psi_{R}$}

Snapshots of $u_{H}$ (see figure $11 \mathrm{~b}$ and d) show that isovalues of this quantity are closely related to isocontours of the streamfunction in the rotating frame of reference $\Psi_{R} \equiv \Psi+\frac{1}{2} r^{2} \Omega$. This indicates that the flow is close to an Euler equilibrium. Indeed, assume a single helical vortex possesses an inviscid equilibrium solution 
(a)

(c)
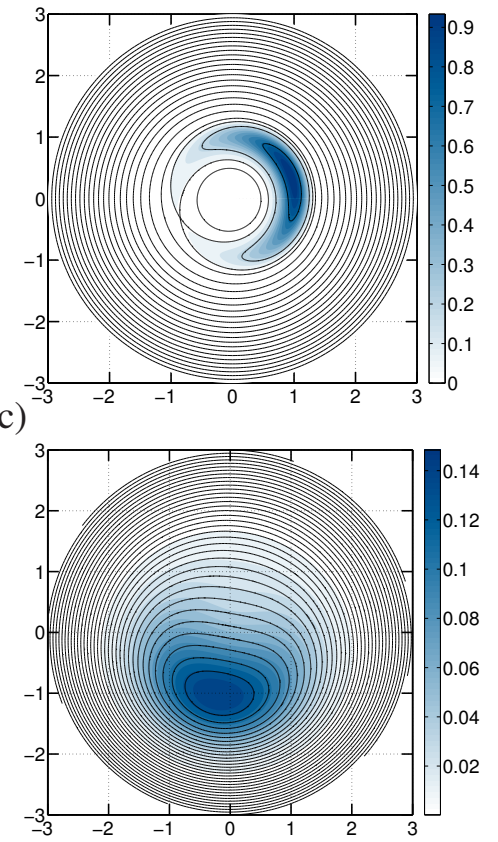

(b)

(d)
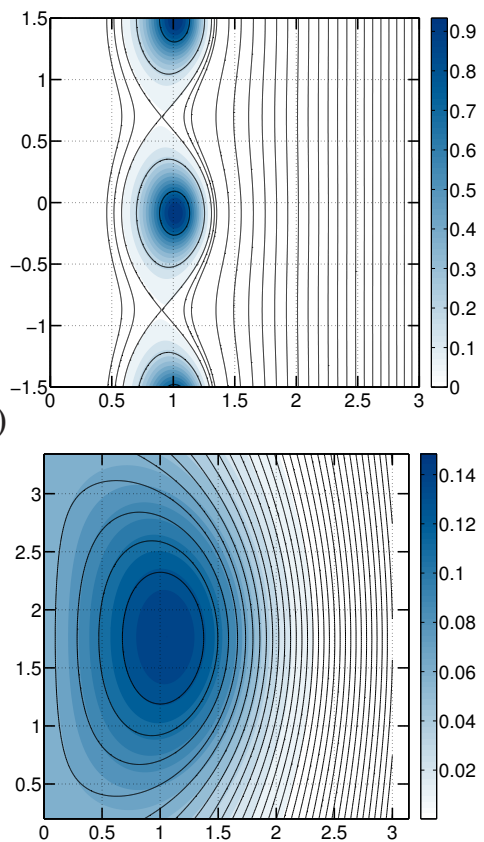

FIG. 10: Helical vortex at $R e=5000$ of reduced pitch (a)-(b) $L=0.25$ at $\tau=92.5$, and (c)-(d) $L=1$ at $\tau=1750$. (a) and (c): isocontours in the $\Pi_{0}$-plane (black lines) of the corotating streamfunction $\Psi_{R} \equiv \Psi+\frac{1}{2} r^{2} \Omega\left(\Omega\right.$ is the angular velocity of the vortex) superimposed on top of quantity $\alpha \omega_{B}$; (b) and (d) representation in the meridional plane $\Pi_{z}$.

rotating at angular velocity $\Omega$ : such a solution is of the form $u_{H}(r, \varphi, t)=u_{H}(r, \varphi-\Omega t)$ so that

$$
\partial_{t} u_{H}=-\Omega \frac{\partial u_{H}}{\partial \varphi} .
$$

Exact inviscid relation (25) then reduces to $J\left(u_{H}, \Psi_{R}\right)=0$, which implies that $u_{H}$ is a univoque function of $\Psi_{R}$ :

$$
u_{H}=F\left(\Psi_{R}\right)
$$

Snapshots of $\alpha \omega_{B}$ (see figures 10 and $11 \mathrm{a}$ and c) show that isovalues of $\alpha \omega_{B}$ are also related to streamlines of $\Psi_{R}$. Again this implies that the flow is close to an Euler equilibrium. For the specific case $u_{H}=0$, inviscid rotating equilibria satisfy $J\left(\alpha \omega_{B}, \Psi_{R}\right)=0$ as implied by equation (27), and this imposes $\alpha \omega_{B}$ to be a univoque function of $\Psi_{R}$ as well:

$$
\alpha \omega_{B}=G\left(\Psi_{R}\right) \text { when } u_{H}=0 .
$$


(a)

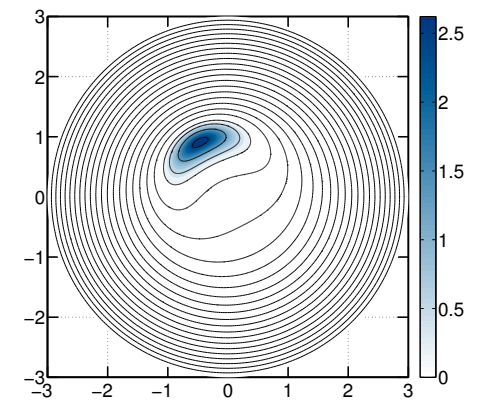

(c)

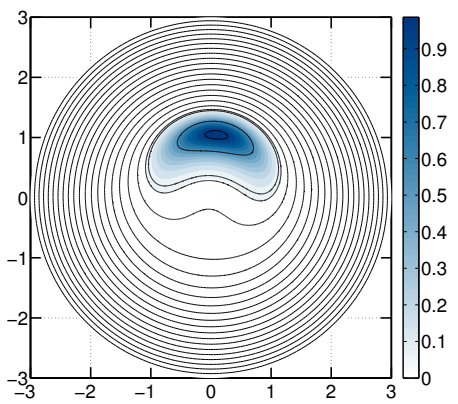

(b)

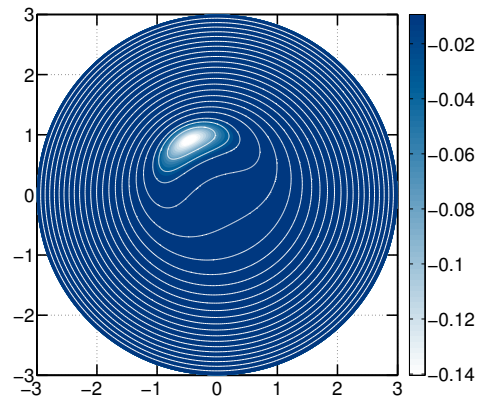

(d)

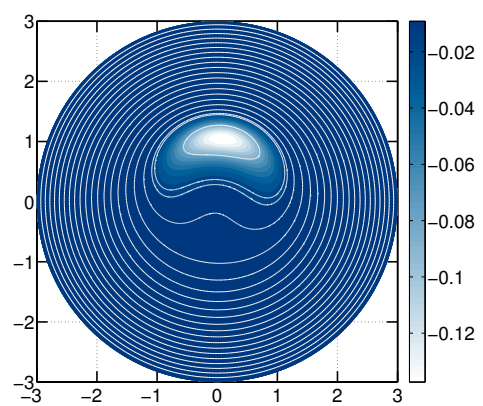

FIG. 11: Helical vortex of pitch $L=0.5$ at $R e=5000$ for times (a)-(b): $\tau=62.5$ and (c)-(d): $\tau=162.5$. (a) and (c): quantity $\alpha \omega_{B}$ (in color) with isocontours (black lines) of $\Psi_{R}$ the corotating streamfunction. (b) and (d): helical velocity $u_{H}$ (in color) with isocontours (white lines) of $\Psi_{R}$. Representation in the $\Pi_{0}$-plane.

This exact relation for $u_{H}=0$ becomes approximate for non-uniform $u_{H}$ distributions. Indeed inviscid equilibria should satisfy in that case equation (26) together with equation (46), yielding

$$
J\left(\alpha \omega_{B}, \Psi_{R}\right)+\frac{2 \alpha^{4}}{L^{2}} u_{H}^{(R)} \frac{\partial u_{H}}{\partial \varphi}=0
$$

where $u_{H}^{(R)}=u_{H}-L \Omega$ is the value of $u_{H}$ in the rotating frame. If the second term in equation (48) is discarded, e.g. when $u_{H}$ is small, one recovers equation (47).

Both conclusions are further confirmed by the scatterplots in figure 12a and b: the points $\left(\alpha \omega_{B}, \Psi_{R}\right)$ and $\left(u_{H}, \alpha \omega_{B}\right)$ are aligned on a single curve for any fixed time. For an Euler flow, the functional relationship would be steady. Here, this state is called quasi-equilibrium since it evolves in time because of viscous diffusion, leading to a slow time dependence of the functional relationship as well. Figure 12b confirms a linear dependence between $u_{H}$ and $\alpha \omega_{B}$ as predicted by equation (34). Such relationships were also verified for the case of two helical vortices during their quasi-equilibrium stage of evolution [27]. 

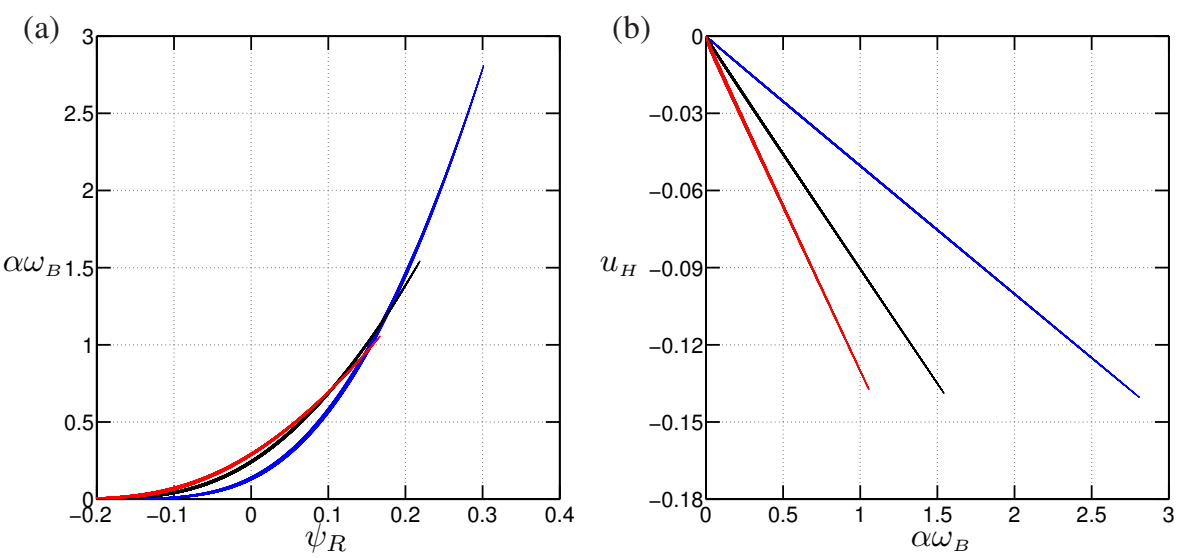

FIG. 12: Helical vortex of reduced pitch $L=0.5$ at $R e=5000$. Scatter plots of (a) $\left(\alpha \omega_{B}, \Psi_{R}\right)$, and (b) $\left(u_{H}, \alpha \omega_{B}\right)$ at $\tau=62.5$ (blue), 112.5 (black), 162.5 (red).
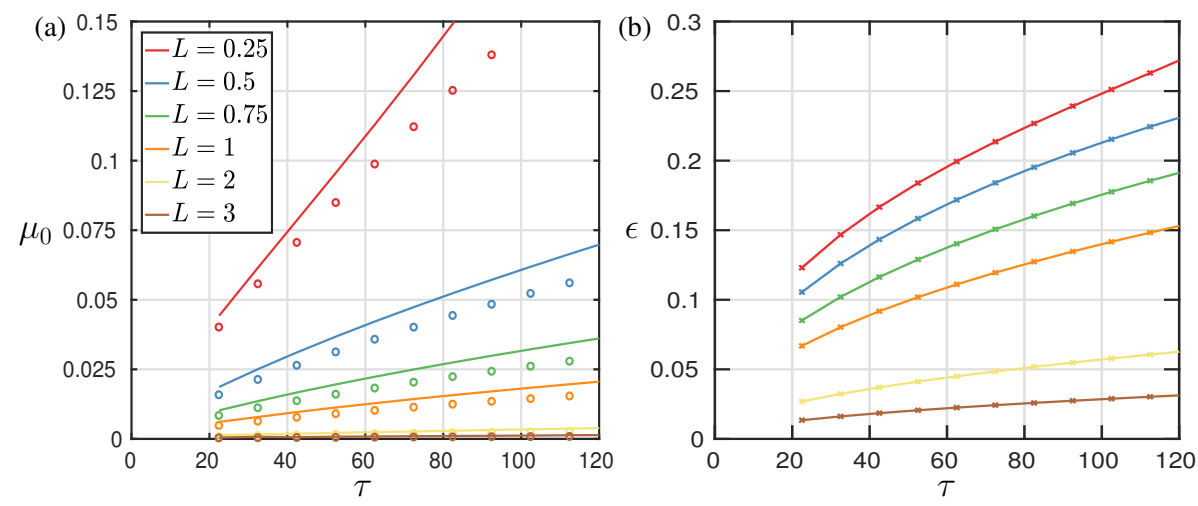

FIG. 13: Helical vortex at $R e=5000$ for different values of $L$ between 0.25 and 3. (a) Time evolution of ellipticity $\mu_{0}$. Solid lines: values determined from the DNS; circles: ellipticity predicted by equation (51).

(b) Time evolution of $\varepsilon$ measured through the DNS.

\section{Strain and ellipticity}

Quasi-equilibrium solutions are not purely axisymmetric (see figures $3 \mathrm{~b}$ and d). In appendix B 3, it is explained how streamline ellipticity $\mu(\rho)$ can be numerically computed. This quantity slightly varies with $\rho$ within the core (see figure 19 in appendix B). In this section, we use the ellipticity in the center of the vortex core $\mu_{0} \equiv \mu(\rho=0)$ as a measure of the core ellipticity and focus hereafter on its time evolution. On figure 13a, the time evolution of the measured ellipticity $\mu_{0}$ is plotted for various values of $L$. As $L$ is decreased, it is found that the ellipticity increases. For a single helical vortex, an asymptotic analysis was 
performed in Ref. [25] providing an expression for the streamfunction near the vortex center (written below with the non-dimensional units used in Ref. [25])

$$
\Psi(\rho, \psi)=-\frac{\rho^{2}}{2}+\varepsilon^{2} S^{(2)} \rho^{2} \cos 2 \psi+\cdots \quad \text { for } \rho \rightarrow 0,
$$

where the parameter $\varepsilon$ is defined as

$$
\varepsilon \equiv \frac{a}{r_{A}} \frac{1}{1+L^{2} / r_{A}^{2}}
$$

and $S^{(2)}$ is a dimensionless quantity. From expression (49), it is easy to compute the ellipticity of streamlines near the vortex center using relation (B10) of appendix B 3 which yields

$$
\mu_{0} \approx-\varepsilon^{2} S^{(2)}
$$

The dimensionless quantity $S^{(2)}$ is the sum of two contributions

$$
S^{(2)}=S_{N H}+2.525 S .
$$

The first term $S_{N H}$ is due to local curvature effects and is computed in Ref. [25]. In the present work we use in our computation the value with zero axial velocity $S_{N H}=-0.2$. The second term is related to a strain rate $2 S$ which is not externally imposed here but self-induced, due the other parts of the vortex. In Ref. [25], the value of $S\left(\varepsilon, L / r_{A}\right)$ for a single helical vortex is explicited in their equation (4.16) as a function of $\varepsilon$ and $L / r_{A}$. Since parameter $\varepsilon$ is assumed small in this asymptotic analysis, the vortex core size has to remain small with respect the radius $r_{A}$ and the reduced pitch $L$. This condition is met here especially at large $L$, as shown in figure $13 \mathrm{~b}$ where the time evolution of $\varepsilon$ is plotted. Note that the case of a two-dimensional vortex with a Gaussian vorticity profile subjected to an external strain field $\gamma$ is recovered from (51)-(52) by setting $S_{N H}=0$ and $\varepsilon^{2} S=\gamma / 2[35]$.

Equations (51) and (52) lead to an analytical model for $\mu_{0}$ which is plotted in figure 13a for various values of $L$. For large $L$, the self-induced strain field is almost null and so is the ellipticity. For small $L$, the agreement is fair, but not close. As mentioned by an anonymous referee, taking into account the angular rotation of single helical vortex may reduce the discrepancy between model and DNS results. Indeed, it is known that rotation affects ellipticity for two-dimensional vortices in an external rotating strain [36]. The asymptotic analysis performed [25] for helical vortices however does not introduce the role of rotation on the two terms of equation (52). Introducing the role of rotation on the curvature and strain terms would necessitate an asymptotic work by itself that we leave for a future study.

\section{E. Flow topology in the rotating frame}

As time evolves, the vortex core size increases, which may induce a modification of the flow topology in the co-rotating frame. In this respect, one may focus on the orbits, i.e. lines of constant $\Psi_{R}$ projected onto the planes $\Pi_{0}$ and $\Pi_{z}$ at each time and, more specifically, to the number and position of critical points. This extends the work of Ref. [23] to nonsingular vorticity distributions and quasi-steady viscous equilibria. In figure 14, the isovalues of $\Psi_{R}$ at fixed pitch $L=0.5$ are plotted in $\Pi_{0}$ (left graphs) for increasing times, thus for increasing core sizes. Without loss of generality, the vortex has been rotated so that its center (red dot) lies at $\theta=0$. For small $a$ (figure 14a), there is only one critical point, corresponding to the vortex center. When $a$ is close to $L$ (figure 14b), an elliptic (blue) and a saddle (green) point now appear, both situated at $\theta=\pi$, 
(a)

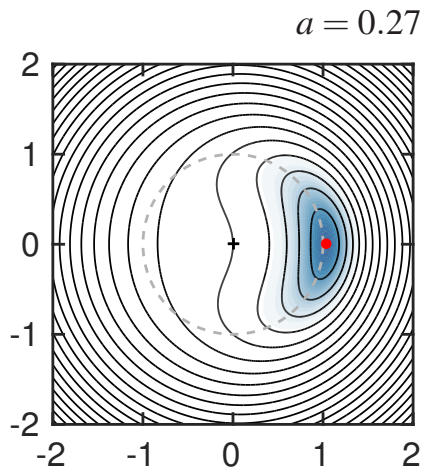

(c)

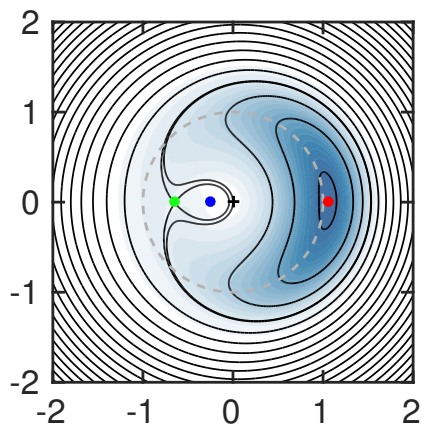

(e)

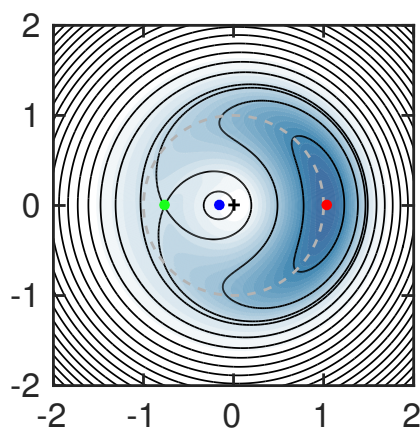

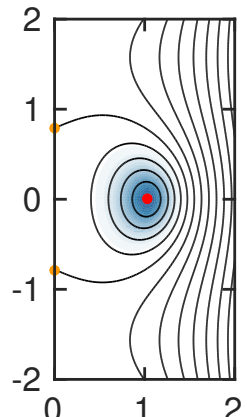
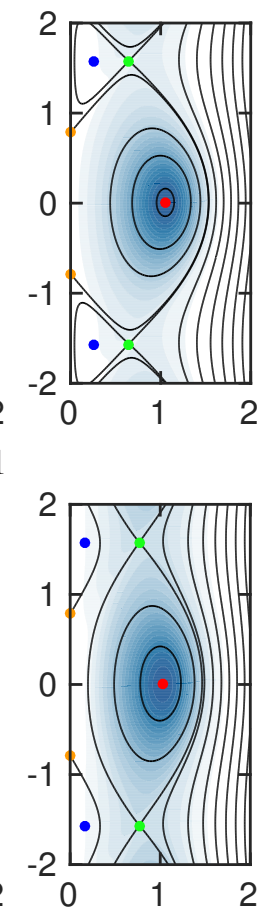

(b)
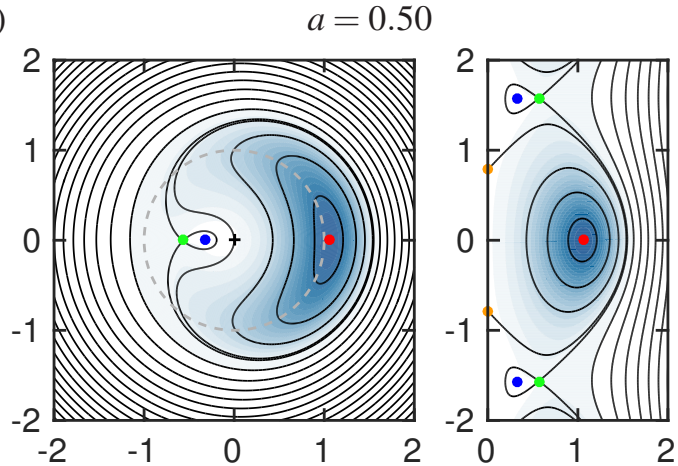

(d)
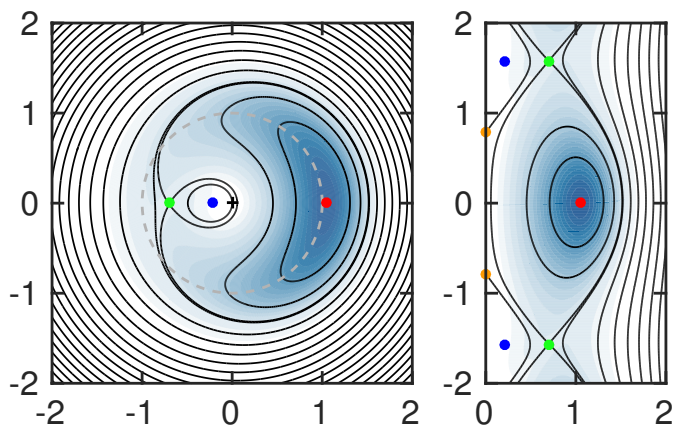

(f)

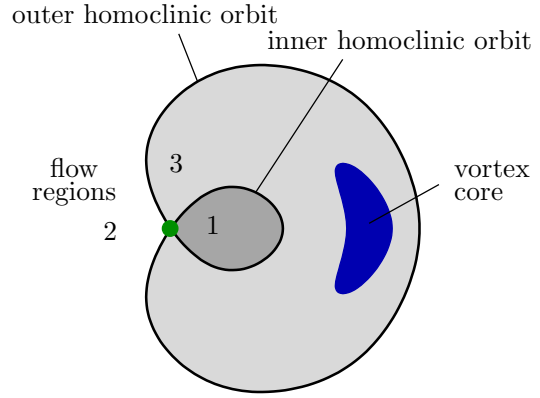

FIG. 14: (a)-(e) Streamline topology in the rotating frame for a helical vortex with different core sizes $a$ at fixed $L=0.5$. For each value of $a$, streamlines are represented in planes $\Pi_{0}$ (left) and $\Pi_{z}$ (right). Critical points are pinpointed as solid circles. (f) Sketch of the three flow regions defined by the homoclinic orbits.

opposite to the vortex center. These points impose a structure to the flow. Based on the homoclinic orbits of the saddle in $\Pi_{0}$ three regions can be defined, as sketched in figure 14f: $(i)$ a region 1 enclosed by the inner homoclinic orbit containing the additional elliptic point, (ii) a region 2 located outside the outer homoclinic orbit and (iii) a region 3 that contains the vortex center and complements the two other regions. These three regions have their counterpart in the plane $\Pi_{z}$ (right graphs in figure 14): the vortex centerline cuts $\Pi_{z}$ at 
$z=(0, \pm 2, \pm 4, \cdots) \pi L$, while elliptic and saddle points are located at $z=( \pm 1, \pm 3, \cdots) \pi L$. Additional points (yellow dots in figure 14) emerge at $r=0$ and $z=\left( \pm \frac{1}{2}, \pm \frac{3}{2}, \cdots\right) \pi L$. They are associated to the streamline in the $\Pi_{0}$ plane passing through the origin. A helical streamtube (region 1) thus appears at a certain distance of the axis. As $a$ is increased, the elliptic and saddle point progressively move apart radially, which increases the area of region 1. The topology is modified when $a$ crosses the value 0.54 (see figures $14 \mathrm{c}$ and d): region 1 in the plane $\Pi_{0}$ now contains the origin, and, more importantly, in the $\Pi_{z}$ plane, region 3 gets away from the $z$-axis which is now fully contained in region 1 . When $a / L$ is large enough, region 3 tends to form a cylindrical shear layer, which consequently induces a jet/wake near the axis, that is in region 1 .

(a)

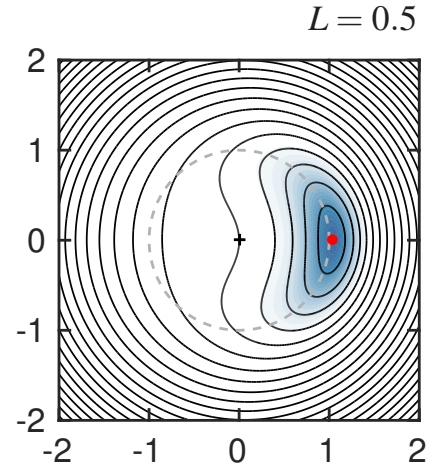

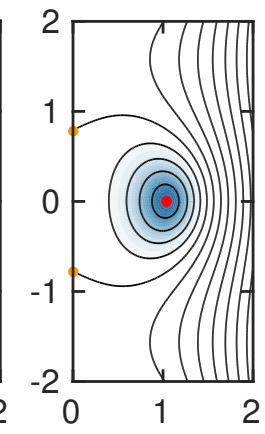

(b)

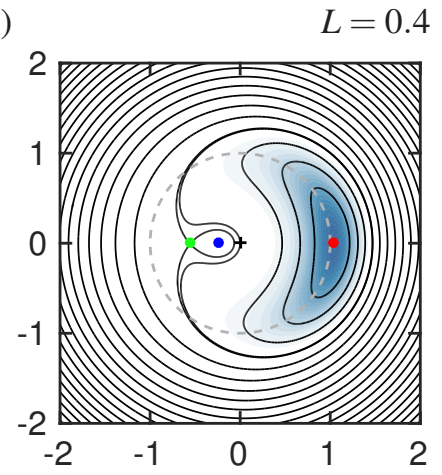

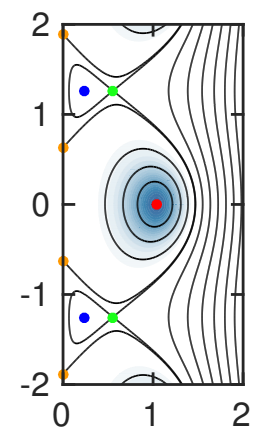

(c)
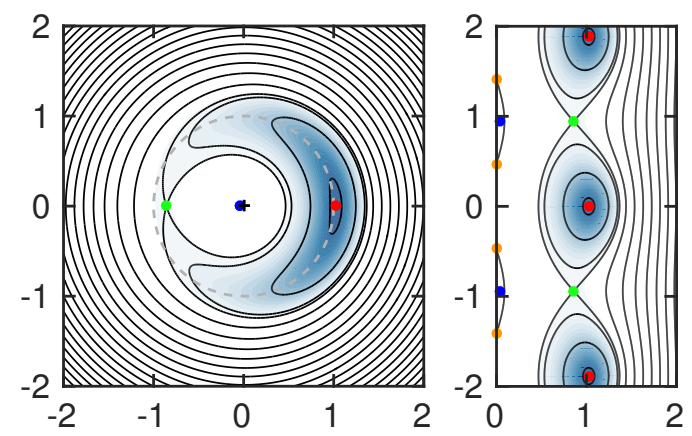

FIG. 15: Rotating frame: streamline topology for one helical vortex of core size $a=0.3$ and for different $L$. Representation in planes $\Pi_{0}$ (left) and $\Pi_{z}$ (right). Critical points are pinpointed as solid circles.

When $L$ is decreased keeping the core radius $a$ fixed, the streamline topology evolves in a similar manner as the one described when $a$ is increased at fixed $L$. This is shown in figure 15, where iso-contours of $\Psi_{R}$ are plotted for $a=0.3$ and different pitches $L=0.5,0.4,0.3$.

In order to better characterize the flow topology at each time, one may also provide three-dimensional Lagrangian orbits in the frozen flow corresponding to the quasi-steady state at that time. This is performed by time-integrating the true three-dimensional dynamics of Lagrangian particles in the reference frame rotating with the vortex. Note that these orbits are bound to stick to helical surfaces of constant $\Psi_{R}$, but they are not themselves helically symmetric.

Hereafter, we examine such orbits for the three cases listed in table I. 


\begin{tabular}{ccc}
\hline case & $L$ & $a$ \\
\hline $\mathrm{a}$ & 0.5 & 0.06 \\
$\mathrm{~b}$ & 0.5 & 0.25 \\
$\mathrm{c}$ & 0.25 & 0.25 \\
\hline
\end{tabular}

TABLE I: Case definition for the study of Lagrangian orbits.

For cases a and b, the flow topology is similar to that of figure 15a, while for case c, additional critical points are present as in figure 15c. For each case, three different trajectories have been considered (see figure 16): in the vicinity of the vortex core (left), in the vicinity of the $z$-axis (center), and in the outer potential region (right). Orbits initiated near the vortex core loop around the vortex with a spatial periodicity which depends on the core size, the pitch and their distance to the vortex core center. When initiated in the outer potential region, the orbits are "modulated" helical trajectories with small pitch, which are weakly influenced by the geometrical parameters of the vortex. When initiated near the $z$-axis, two scenarios occur. For cases a and $b$ (see figures 16a and $\mathrm{b}$ center graph), there is no critical point, and orbits alternatively loop around the vortex core and progress along a path in the vicinity of the $z$-axis. For case $\mathrm{c}$ (see figure 16c center graph), orbits are quenched between the critical points hence in the vicinity of the $z$ axis. The emergence of such orbits in the inner region is to be linked to the fact that, at smaller $L$ values, the vorticity distribution more and more resembles a cylindrical vortex sheet, which is known to induce an axial velocity component inside the cylinder.

\section{CONCLUDING REMARKS}

Quasi-equilibria of helical vortex systems have been numerically investigated in the helical symmetry context. In particular, generic quasi-equilibrium state of one helical vortex has been obtained stemming from a singular helical line vortex. Accurate tools were developed for characterization showing several features. First the helical vorticity component in the plane locally orthogonal to the vortex centerline is found to be self-similar and to have a Gaussian distribution with radius $a(\tau) \approx(4 \tau / R e)^{1 / 2}$ and decaying amplitude $1 /\left(\pi a^{2}(\tau)\right)$. Second the helical velocity component is coupled to the helical vorticity through viscous effects leading to a relationship $u_{H}=2 \tau \alpha \omega_{B} /(L R e)$. Consequently a small Gaussian velocity deficit occurs along helical lines and radially spreads at the same rate as vorticity but with a roughly constant amplitude $-\left[2 \pi\left(1+L^{2}\right)\right]^{-1}$. The helical vortex locally endows the profile of a Batchelor vortex. In addition the ratio $q(\tau) \approx-\left(1+L^{2}\right) / a(\tau)$ between the typical azimuthal velocity $1 /(2 \pi a)$ and the constant helical velocity amplitude takes large negative values since the vortex core is assumed small. The helix radius also increases in time but no simple argument was found to explain this precise behaviour. Finally we analyzed the flow topology in the co-rotating frame. To do so, we presented the structure of the helical streamfunction, more precisely the emergence of critical points, as well as three-dimensional Lagrangian orbits. The above results could be potentially extended to arrays of multiple helical vortices, as partially done in [27].

For the specific value $L=1$, and presumably for pitches in the vicinity of this value, a quasi-equilibrium could not be reached (see figure $9 \mathrm{~b}$ ): such state seems to be unstable with respect to perturbations with helical symmetry. A similar behaviour was previously reported for a helical patch [24]. Whether both observations are linked or not is left for future investigations. 
(a)
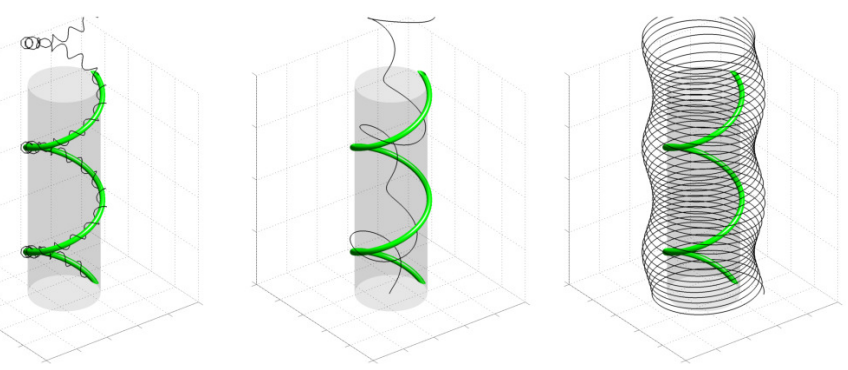

(b)
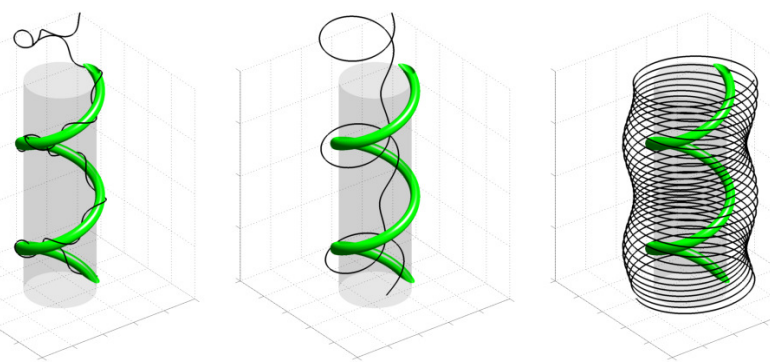

(c)
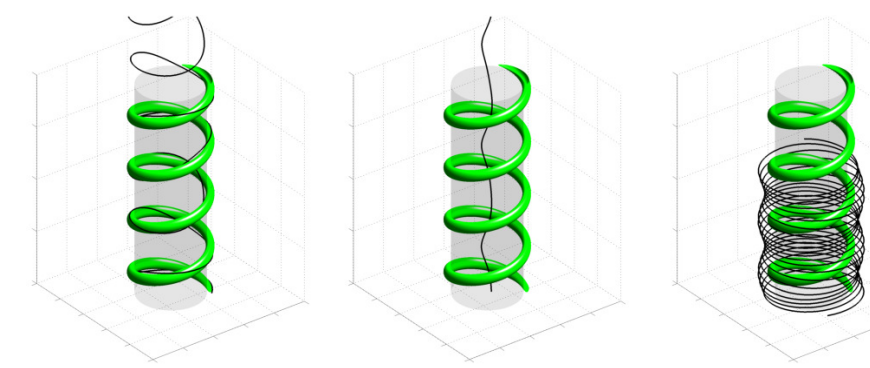

FIG. 16: Lagragian orbits (solid black lines) in the co-rotating frame, around a helical vortex (green tube) for case (a) $L=0.5, a=0.06$, (b) $L=0.5, a=0.25$, (c) $L=0.25, a=0.25$. The orbit is initiated in the vicinity of the vortex core (left), in the vicinity of the $z$-axis (center), and in the potential outer region (right).

\section{ACKNOWLEDGMENTS}

This work received support from the French Agence Nationale de la Recherche under the ANR HELIX project ANR-12-BS09-0023-01. HPC resources from GENCI-IDRIS (grants 2015-2a1386 and 20162a1386) are also acknowledged, as well as technical help from Jalel Chergui, Olivier Lassalle and JeanClaude Barbet at LIMSI. 
Appendix A: Retrieving quantities in plane $\Pi_{\perp}$ from $\Pi_{0}$

This appendix explains how quantities needeed in plane $\Pi_{\perp}$ for vortex characterization are retrieved from numerical data provided in plane $\Pi_{0}$. Since scalars and vortex components are invariant along lines of constant $\varphi$, this amounts to locate, for any point $\mathrm{M}$ of Cartesian coordinates $(\xi, \eta)$ in plane $\Pi_{\perp}$, the point $\mathrm{M}_{0}$ in the plane $\Pi_{0}$ situated on the same helical line than $\mathrm{M}$, as depicted in figure 2 . In the cylindrical frame, the coordinates of $\mathrm{M}$ and $\mathrm{M}_{0}$ are denoted as $\left(r_{M}, \theta_{M}, z_{M}\right)$ and $\left(r_{M_{0}}, \theta_{M_{0}}, 0\right)$ respectively. The position vector $\boldsymbol{O M}$ is first expressed through two different ways, namely from the equation of the helical line:

$$
\boldsymbol{O M}=z_{M} \boldsymbol{e}_{z}+r_{M} \cos \theta_{M} \boldsymbol{e}_{x}+r_{M} \sin \theta_{M} \boldsymbol{e}_{y}
$$

where

$$
\theta_{M}=\theta_{M_{0}}+z_{M} / L \text { and } r_{M}=r_{M_{0}},
$$

or by inserting the vortex center A:

$$
\boldsymbol{O M}=\boldsymbol{O A}+\boldsymbol{\xi} \boldsymbol{e}_{r_{A}}+\eta \boldsymbol{e}_{\varphi_{A}}=\left(r_{A}+\xi\right) \boldsymbol{e}_{r_{A}}+\eta \boldsymbol{e}_{\varphi_{A}}
$$

The height $z_{M}$ can readily be determined:

$$
z_{M}=\boldsymbol{O M} \cdot \boldsymbol{e}_{z}=-\alpha_{A} \frac{r_{A}}{L} \eta, \quad \text { with } \alpha_{A} \equiv\left(1+\frac{r_{A}^{2}}{L^{2}}\right)^{-1 / 2}
$$

so that

$$
r_{M} \boldsymbol{e}_{r}=\boldsymbol{O M}-z_{M} \boldsymbol{e}_{z}=\left(r_{A}+\xi\right) \boldsymbol{e}_{r_{A}}+\eta \alpha_{A}^{2} \boldsymbol{e}_{\varphi_{A}}+\eta \alpha_{A}^{2} \frac{r_{A}}{L} \boldsymbol{e}_{B_{A}},
$$

and, after some straightforward algebra:

$$
\begin{aligned}
r_{M} & =\left[\left(r_{A}+\xi\right)^{2}+\alpha_{A}^{2} \eta^{2}\right]^{1 / 2}, \\
r_{M} \cos \theta_{M} & =\left(r_{A}+\xi\right) \cos \theta_{A}-\eta \alpha_{A} \sin \theta_{A} \\
r_{M} \sin \theta_{M} & =\left(r_{A}+\xi\right) \sin \theta_{A}+\eta \alpha_{A} \cos \theta_{A} .
\end{aligned}
$$

For vortex characterization, a regular polar mesh with nodes $\left(\rho_{p}, \psi_{q}\right)$ is created in plane $\Pi_{\perp}$. Relations (A6)-(A8) with $\xi=\rho_{p} \cos \psi_{q}$ and $\eta=\rho_{p} \sin \psi_{q}$ lead to determine $r_{M}$ and $\theta_{M}$ which, together with (A2), give access to the coordinates of the image points $\mathrm{M}_{0}$ in $\Pi_{0}$. Since these latter points do not coincide with the nodes at which the numerical data are provided, it is found necessary to use a 5th-order accurate interpolation based on Tchebychev polynomials. This is performed for all vorticity and velocity components. The in-plane vorticity components $\omega_{\rho}=\boldsymbol{\omega} \cdot \boldsymbol{e}_{\rho}$ and $\omega_{\psi}=\boldsymbol{\omega} \cdot \boldsymbol{e}_{\psi}$ as well as the component $\omega_{B_{A}}=\boldsymbol{\omega} \cdot \boldsymbol{e}_{B_{A}}$ normal to $\Pi_{\perp}$ are then determined, with $\left(\boldsymbol{e}_{\rho}, \boldsymbol{e}_{\psi}\right)$ being the local polar basis in $\Pi_{\perp}$. Using the components of $\boldsymbol{e}_{B_{A}}, \boldsymbol{e}_{\rho}$ and $\boldsymbol{e}_{\psi}$ 
on the basis $\left(\boldsymbol{e}_{B}, \boldsymbol{e}_{\varphi}, \boldsymbol{e}_{r}\right)$ eventually leads to the following expressions

$$
\begin{gathered}
\omega_{B_{A}}=\omega_{B} \alpha_{M} \alpha_{A}\left[1+\frac{r_{M} r_{A}}{L^{2}} \cos \left(\theta_{M}-\theta_{A}\right)\right] \\
+\omega_{\varphi} \alpha_{M} \alpha_{A}\left[\frac{r_{A}}{L} \cos \left(\theta_{M}-\theta_{A}\right)-\frac{r_{M}}{L}\right] \\
+\omega_{r} \alpha_{A} \frac{r_{A}}{L} \sin \left(\theta_{M}-\theta_{A}\right), \\
\omega_{\rho}=\omega_{B}\left[\frac{\alpha_{M} r_{M}}{L} \sin \left(\theta_{A}-\theta_{M}\right) \cos \psi+\alpha_{M} \alpha_{A} \sin \psi\left(-\frac{r_{A}}{L}+\frac{r_{M}}{L} \cos \left(\theta_{M}-\theta_{A}\right)\right)\right] \\
+\omega_{r}\left[\cos \left(\theta_{M}-\theta_{A}\right) \cos \psi+\alpha_{A} \sin \left(\theta_{M}-\theta_{A}\right) \sin \psi\right] \\
+\omega_{\varphi}\left[\alpha_{M} \sin \left(\theta_{A}-\theta_{M}\right) \cos \psi+\alpha_{M} \alpha_{A} \frac{r_{M} r_{A}}{L^{2}}+\cos \left(\theta_{M}-\theta_{A}\right) \sin \psi\right], \\
\omega_{\psi}=\omega_{B}\left[-\frac{\alpha_{M} r_{M}}{L} \sin \left(\theta_{A}-\theta_{M}\right) \sin \psi+\alpha_{M} \alpha_{A} \cos \psi\left(-\frac{r_{A}}{L}+\frac{r_{M}}{L} \cos \left(\theta_{M}-\theta_{A}\right)\right)\right] \\
+\omega_{r}\left[-\cos \left(\theta_{M}-\theta_{A}\right) \sin \psi+\alpha_{A} \sin \left(\theta_{M}-\theta_{A}\right) \cos \psi\right] \\
+\omega_{\varphi}\left[-\alpha_{M} \sin \left(\theta_{A}-\theta_{M}\right) \sin \psi+\alpha_{M} \alpha_{A} \frac{r_{M} r_{A}}{L^{2}}+\cos \left(\theta_{M}-\theta_{A}\right) \cos \psi\right] .
\end{gathered}
$$

\section{Appendix B: Vortex characterization}

This appendix explains the specific characterization techniques used to determine helix radius, angular velocity, core radius and ellipticity.

\section{Helix radius $r_{A}$ and angular velocity $\Omega$}

In the plane $\Pi_{0}$, the precise value of the helix radius $r_{A}$ is obtained using a local quadratic interpolation of $\omega_{B}$ around the mesh point of maximum $\omega_{B}\left(r_{i}, \theta_{j}\right)$, from which the coordinates $\left(r_{A}, \theta_{A}\right)$ of the maximum of the interpolating paraboloid can be accurately deduced.

Configurations of one helical vortex or a regular array of identical vortices may be inviscid equilibria when considered in a frame rotating at some constant angular velocity $\Omega$. This rotation is due to both the selfinduced vortex velocity and the mutual induction between vortices. When viscous diffusion acts, it gives rise to a slowly evolving state that we call quasi-equilibrium with changing angular velocity, helix radius and core size. A first method for evaluating the instantaneous angular velocity $\Omega(t)$ is to track the azimuthal location of the vortex center $\theta_{A}^{-}$and $\theta_{A}^{+}$for times $t_{-}$and $t_{+}$respectively and to set $\Omega(t)=\left(\theta_{A}^{+}-\theta_{A}^{-}\right) /\left(t_{+}-t_{-}\right)$at time $t=\frac{1}{2}\left(t_{-}+t_{+}\right)$. Such a procedure is inaccurate because determining $\theta_{A}^{ \pm}$may be quite sensitive to the actual position of the vortex center within the numerical cell. Instead we use the vorticity component $\omega_{B}$ in the whole plane $\Pi_{0}$ at times $t_{-}$and $t_{+}$. The rotation angle $\delta \theta$ of the vortex along the azimuth between times $t_{-}$and $t_{+}$is determined so as to achieve the best correlation between $\omega_{B}\left(r, \theta+\delta \theta, t_{-}\right)$and $\omega_{B}\left(r, \theta, t_{+}\right)$. Technically, the positive integral

$$
I(\delta \theta) \equiv \iint_{S}\left|\omega_{B}\left(r, \theta, t_{+}\right)-\omega_{B}\left(r, \theta+\delta \theta, t_{-}\right)\right|^{2} \mathrm{~d} S
$$


is minimized with respect to $\delta \theta$ using an iterative procedure (note that quantity $\omega_{B}\left(r, \theta+\delta \theta, t_{-}\right.$) is accurately obtained from $\omega_{B}\left(r, \theta, t_{-}\right)$by simple multiplications of azimuthal modes $\omega_{B}^{(m)}$ by $\left.\mathrm{e}^{\mathrm{i} m \delta \theta}\right)$. This approach is justified since for inviscid equilibria, $I(\delta \theta)$ vanishes for $\delta \theta=\left(t_{+}-t_{-}\right) \Omega$. For viscous quasiequilibria, $I(\delta \theta)$ is assumed to reach a minimum when $\delta \theta=\left(t_{+}-t_{-}\right) \Omega(t)$, which yields $\Omega(t)$ for $t=$ $\frac{1}{2}\left(t_{-}+t_{+}\right)$.

\section{Vortex core radius}

In order to evaluate the core size, we focus on the axisymmetric part of expansion (13) in the plane $\Pi_{\perp}$. A Gaussian fit is assumed for the monopolar component $\omega_{B_{A}}^{(0)}$ :

$$
f(\rho ; C, a)=C \mathrm{e}^{-(\rho / a)^{2}},
$$

and parameters $C$ and $a$ are evaluated so that distribution (B2) achieves the best fit on the interval $\left[0, \rho_{\text {cut }}\right]$ in which vorticity is significant (see below for a discussion on the appropriate values of $\rho_{\text {cut }}$ ). Quantity $a$ quantifies the vortex core radius.
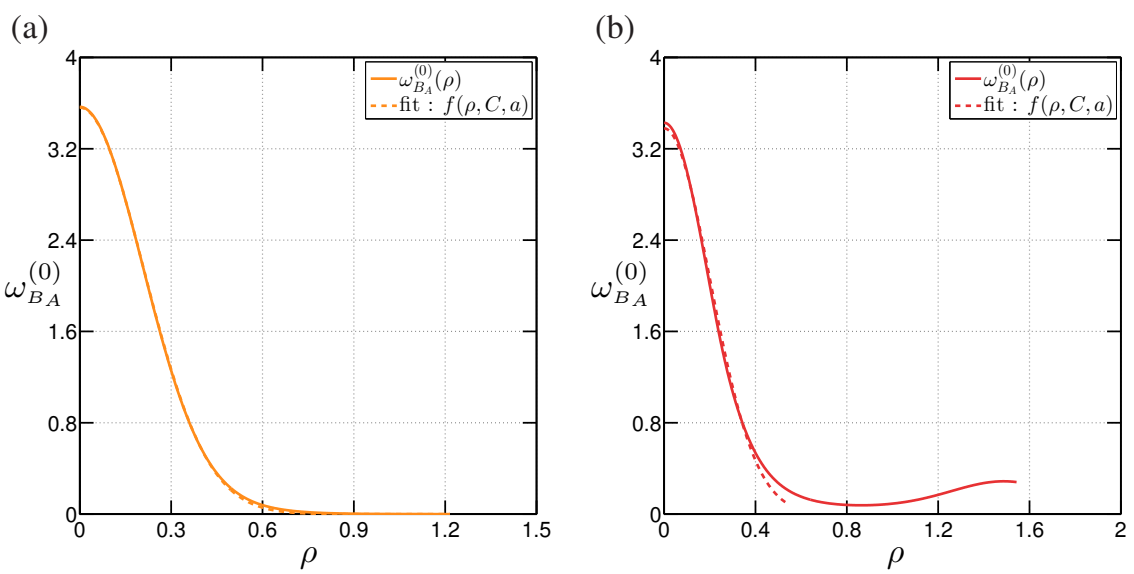

FIG. 17: Helical vortex of reduced pitch (a) $L=1$ and (b) $L=0.25$ : monopolar vorticity component $\omega_{B_{A}}^{(0)}(\rho)$ (continuous line) and its Gaussian fit (dashed line).

The Gaussian profile is an excellent fit for a helical vortex of pitch $L=1$, as can be seen on figure 17a. For lower pitches (see figure $17 \mathrm{~b}$ at $L=0.25$ ), the fit deteriorates at the periphery of the vortex. For smaller values of $L$ (typically when $L<0.5$ ), the plane $\Pi_{\perp}$ cuts several adjacent turns of the vortex (see figure $3 \mathrm{~b}$ ). As a consequence, the vorticity of adjacent turns may overlap. This means that $\rho_{\text {cut }}$ should thus be chosen such that contributions due to adjacent turns are not taken into account, that is $\rho_{\text {cut }}$ cannot exceed half the spacing between turns $\pi L d$, where factor $d=R / \sqrt{R^{2}+L^{2}}$ arises from the angle between planes $\Pi_{\perp}$ and $\Pi_{z}$. In addition, $\rho_{\text {cut }}$ cannot be smaller that $2 a$ so that most part of vorticity distribution be fitted. This imposes a major constraint, especially when the core size $a$ is large: $2 a \leq \rho_{\text {cut }} \leq \pi L d$. For the case $L=0.25$ in figure $17 \mathrm{~b}$, one has $2 a \approx 0.6, \rho_{\text {cut }}=0.6$ and $\pi L d=0.75$. For large $L$, the plane $\Pi_{\perp}$ intercepts the helical 
vortex only once (figure 17a), thus the evaluation of the core radius is not sensitive to $\rho_{\text {cut }}$ if large enough. Typically, $\rho_{\text {cut }}$ is chosen larger than $4 a$ when $L \geq 0.5$.

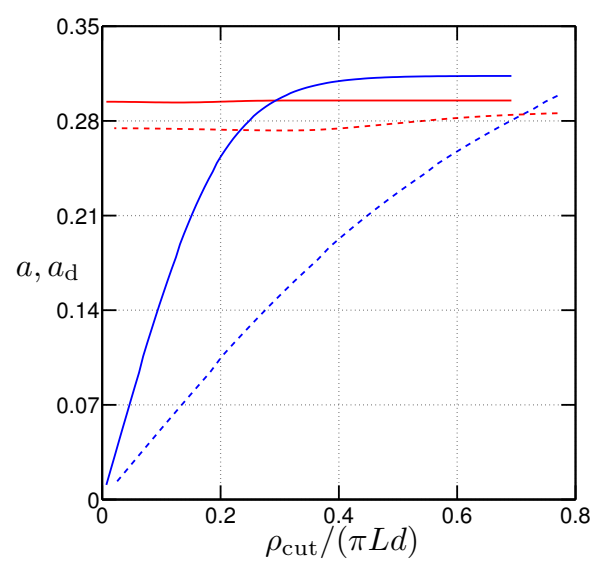

FIG. 18: Helical vortex of reduced pitch $L=1$ (solid) and $L=0.25$ (dashed). Fitted core size $a$ (top curves in red) and dispersion radius $a_{\mathrm{d}}$ (blue) as functions of $\rho_{\text {cut }} /(\pi L d)$. The fitted core size $a$ is found much less sensitive to $\rho_{\text {cut }}$ than the dispersion radius.

Note that the definition of the core size is not unique: for two-dimensional vortices, moments of vorticity are commonly used. In analogy with the two-dimensional case, one could alternatively employ the vorticity moments of $\omega_{B_{A}}(\rho, \psi)$ about the center point $\mathrm{A}$ in the $\Pi_{\perp}$ plane:

$$
a_{\mathrm{d}}^{2}=\frac{\iint \rho^{2} \omega_{B_{A}}(\rho, \psi) \rho \mathrm{d} \rho \mathrm{d} \psi}{\iint \omega_{B_{A}}(\rho, \psi) \rho \mathrm{d} \rho \mathrm{d} \psi}=\frac{\int_{0}^{\rho_{\mathrm{cut}}} \rho^{3} \omega_{B_{A}}^{(0)}(\rho) \mathrm{d} \rho}{\int_{0}^{\rho_{\mathrm{cut}}} \rho \omega_{B_{A}}^{(0)}(\rho) \mathrm{d} \rho} .
$$

This definition, however, is very sensitive to the presence of vorticity far from the center because of the $\rho^{3}$ factor in the integral. It is shown that, especially at low $L$, the results for $a_{\mathrm{d}}$ strongly depend on the cutoff radius $\rho_{\text {cut }}$, as depicted in figure 18 . This makes the core size definition based on vorticity moments inadequate for helical vortices.

\section{Vortex core ellipticity}

In figures $3 b$ and $d$, it is seen that as one moves away from the vortex center A, i.e. as $\rho$ is increased, vorticity contours are shifting their center towards the left along $\boldsymbol{e}_{r_{A}}$ and are also changing their ellipticity with a major axis directed along $\boldsymbol{e}_{\varphi_{A}}$ and a minor axis directed along $\boldsymbol{e}_{r_{A}}$. This is coherent with the asymptotic analysis performed in [25] in which the streamfunction $\Psi_{R}$ in the co-rotating frame is assumed to be the expanded in term of $\varepsilon$ defined in equation (50). At leading order $\varepsilon^{0}$, the structure is monopolar, i.e. a local columnar axisymmetrical vortex. The main dipolar correction which induces the center shift towards the center of curvature of the filament, i.e. along $\boldsymbol{e}_{r_{A}}$, arises at first order in $\varepsilon$. Quadrupolar corrections quantify the 
ellipticity of the streamlines, i.e. the elliptical deformation of the vortex under the effect of strain. They are generated as well as other dipolar and monopolar corrections, at second order in $\varepsilon^{2}$. Focusing on quadrupolar and dipolar corrections at lower orders, the expansion in [25] implies that

$$
\Psi_{R}(\rho, \psi)=\Psi_{R}^{(0)}(\rho)+\varepsilon \Psi_{R}^{(1)}(\rho) \cos \psi+\varepsilon^{2} \Psi_{R}^{(2)}(\rho) \cos 2 \psi+\cdots
$$

We extend the methodology used in [35] to evaluate the shape of streamlines in the co-rotating reference frame. From the above expansion, one may assume that streamline of level $\Psi_{R}$ is located at

$$
\rho=\rho^{(0)}+\varepsilon \rho^{(1)} \cos \psi+\varepsilon^{2} \rho^{(2)} \cos 2 \psi+\cdots
$$

where the real quantities $\rho^{(0)}, \rho^{(1)}$ and $\rho^{(2)}$ depend only on $\Psi_{R}$. If higher multipolar contributions are discarded in (B5), this corresponds to an ellipse with a center shifted of $\varepsilon \rho^{(1)}$ along the $\boldsymbol{e}_{r_{A}}$ axis with a semi-axis directed along $\boldsymbol{e}_{r_{A}}$ of length $A\left(\rho^{(0)}\right)=\rho^{(0)}+\varepsilon^{2} \rho^{(2)}$ and the second semi-axis directed along $\boldsymbol{e}_{\varphi_{A}}$ of length $B\left(\rho^{(0)}\right)=\rho^{(0)}-\varepsilon^{2} \rho^{(2)}$. Ellipticity is hence

$$
\mu\left(\rho^{(0)}\right)=\frac{|A-B|}{A+B}=\varepsilon^{2} \frac{\left|\rho^{(2)}\right|}{\rho^{(0)}} .
$$

Introducing expansion (B5) in expansion (B4) at order $\varepsilon$, one gets

$$
\Psi_{R}(\rho, \psi)=\Psi_{R}^{(0)}\left(\rho^{(0)}\right)+\varepsilon\left[\Psi_{R}^{(1)}+\rho^{(1)} \frac{\mathrm{d} \Psi_{R}^{(0)}}{\mathrm{d} \rho}\left(\rho^{(0)}\right)\right] \cos \psi+\cdots
$$

leading to a shift

$$
\varepsilon \rho^{(1)}=-\frac{\varepsilon \Psi_{R}^{(1)}\left(\rho^{(0)}\right)}{\frac{\mathrm{d} \Psi_{R}^{(0)}}{\mathrm{d} \rho}\left(\rho^{(0)}\right)} \text { evaluated at } \rho^{(0)}
$$

At order $\varepsilon^{2}$, putting to zero the term in $\cos 2 \psi$, one gets the relation

$$
\rho^{(2)}=-\frac{\Psi_{R}^{(2)}}{\frac{\mathrm{d} \Psi_{R}^{(0)}}{\mathrm{d} \rho}}+\frac{1}{2} \frac{\Psi_{R}^{(1)}}{\left[\frac{\mathrm{d} \Psi_{R}^{(0)}}{\mathrm{d} \rho}\right]^{2}}\left(\frac{\mathrm{d} \Psi_{R}^{(1)}}{\mathrm{d} \rho}-\frac{\Psi_{R}^{(1)}}{2} \frac{d^{2} \Psi_{R}^{(0)}}{d \rho^{2}}\right) \text { evaluated at } \rho^{(0)}
$$

As an example, let us consider a helical vortex at pitches $L=1$ and $L=0.25$. Figure 19a displays the shift of the center of elliptical streamlines as a function of $\rho^{(0)} / a$. From the negative sign of $\rho^{(1)}$ it is confirmed that the center shifts towards the center of curvature as $\rho^{(0)}$ increases. In figure $19 \mathrm{~b}$, the ellipticity $\mu$ is plotted as a function of $\rho^{(0)} / a$. It is found that $\mu$ slightly varies with $\rho^{(0)}$ and is found to increase with radial distance, as expected since vorticity levels gradually decrease. In the paper, quantity $\mu_{0} \equiv \mu(0)$ is used as a measure of the core ellipticity. It is obtained from (B6) and (B9) using l'Hôpital's rule twice, yielding

$$
\mu_{0}=\frac{\varepsilon^{2}}{2}\left|\frac{\mathrm{d}^{2} \Psi_{R}^{(2)}}{\mathrm{d} \rho^{2}}\right| /\left|\frac{\mathrm{d}^{2} \Psi_{R}^{(0)}}{\mathrm{d} \rho^{2}}\right| \quad \text { evaluated at } \rho=0 .
$$



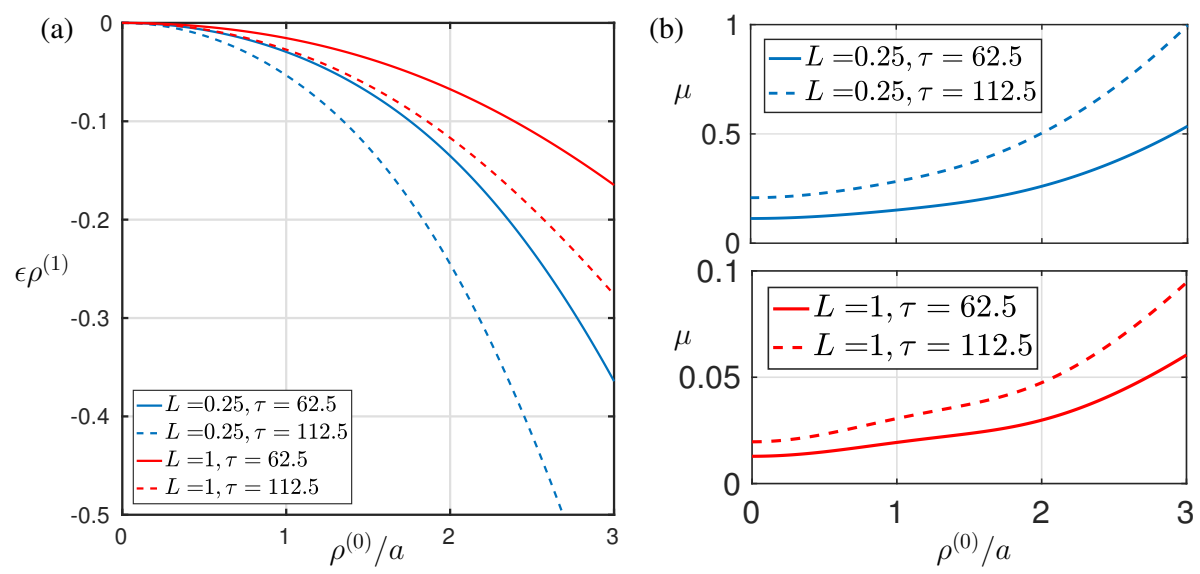

FIG. 19: Helical vortex for $L=0.25$ and $L=1$ at $R e=5000$. Two different core radii are considered: $a=0.22$ corresponding to $\tau=62.5$, and $a=0.30$ to $\tau=112.5$. (a) Shift $\varepsilon \rho^{(1)}$ of elliptical streamlines as a function of $\rho^{(0)} / a$. (b) Ellipticity $\mu$ as a function of $\rho^{(0)} / a$.

From the negative sign of $\rho^{(2)}$, is is seen that the major axis of elliptical streamlines is aligned with the vector $\boldsymbol{e}_{\varphi_{A}}$, hence orthogonal to the $r$-direction.

[1] P. Alfredsson and J. A. Dahlberg, A preliminary wind tunnel study of windmill wake dispersion in various flow conditions, Tech. Rep. FFA TN AU-1499, Part 7(1) (Aeronautical Research Institute of Sweden, 1979).

[2] J. G. Leishman, M. J. Bhagwat, and A. Bagai, "Free-vortex filament methods for the analysis of helicopter rotor wakes," Journal of Aircraft 39, 759-775 (2002).

[3] L. J. Vermeer, J. N. Sørensen, and A. Crespo, “Wind turbine wake aerodynamics,” Prog. Aerosp. Sci. 39, 467-510 (2003).

[4] S. Ivanell, R. Mikkelsen, J. N. Sørensen, and D. Henningson, "Stability analysis of the tip vortices of a wind turbine," Wind Energy 13, 705-715 (2010).

[5] M. Felli, R. Camussi, and F. Di Felice, "Mechanisms of evolution of the propeller wake in the transition and far fields," J. Fluid Mech. 682, 5-53 (2011).

[6] M. Sherry, A. Nemes, D. Lo Jacono, H. M. Blackburn, and J. Sheridan, "The interaction of helical tip and root vortices in a wind turbine wake," Phys. Fluids 25, 117102 (2013).

[7] M. Ali and M. Abid, "Self-similar behaviour of a rotor wake vortex core," J. Fluid Mech. 740 (2014).

[8] S. Sarmast, R. Dadfar, R. F. Mikkelsen, P. Schlatter, S. Ivanell, J. N. Sørensen, and D. S. Henningson, "Mutual inductance instability of the tip vortices behind a wind turbine," J. Fluid Mech. 755, 705-731 (2014).

[9] F. Viola, G. V. Iungo, S. Camarri, F. Porté-Agel, and F. Gallaire, "Prediction of the hub vortex instability in a wind turbine wake: stability analysis with eddy-viscosity models calibrated on wind tunnel data," J. Fluid Mech. 750, R1 (2014).

[10] H. U. Quaranta, H. Bolnot, and T. Leweke, "Long-wave instability of a helical vortex," J. Fluid Mech. 780, 687$716(2015)$.

[11] D. G. Dritschel, "Generalized helical Beltrami flows in hydrodynamics and magnetohydrodynamics," J. Fluid Mech. 222, 525-541 (1991). 
[12] R. L. Ricca, "The effect of torsion on the motion of a helical vortex filament," J. Fluid Mech. 273, 241-259 (1994).

[13] P. A. Kuibin and V. L. Okulov, "Self-induced motion and asymptotic expansion of the velocity field in the vicinity of a helical vortex filament," Phys. Fluids 10, 607 (1998).

[14] D. H. Wood and J. Boersma, "On the motion of multiple helical vortices," J. Fluid Mech. 447, 149-171 (2001).

[15] Y. Fukumoto and V. L. Okulov, "The velocity field induced by a helical vortex tube," Phys. Fluids 17, 107101 (2005).

[16] S. E. Widnall, "The stability of a helical vortex filament,” J. Fluid Mech. 54, 641-663 (1972).

[17] B. P. Gupta and R. G. Loewy, "Theoretical analysis of the aerodynamic stability of multiple, interdigitated helical vortices," AIAA J. 12, 1381-1387 (1974).

[18] V. L. Okulov, "On the stability of multiple helical vortices,” J. Fluid Mech. 521, 319-342 (2004).

[19] V. L. Okulov and J. N. Sørensen, "Stability of helical tip vortices in a rotor far wake," J. Fluid Mech. 576, 1-25 (2007).

[20] Y. Hattori and Y. Fukumoto, "Short-wavelength stability analysis of a helical vortex tube," Phys. Fluids 21, 014104 (2009).

[21] Y. Hattori and Y. Fukumoto, "Modal stability analysis of a helical vortex tube with axial flow," J. Fluid Mech. 738, 222-249 (2014).

[22] F. J. Blanco-Rodríguez and S. Le Dizès, "Elliptic instability of a curved Batchelor vortex,” J. Fluid Mech. 804, 224-247 (2016).

[23] M. Andersen and M. Brøns, “Topology of helical fluid flow,” Eur. J. Appl. Math. 25, 375-396 (2014).

[24] D. Lucas and D. G. Dritschel, "A family of helically symmetric vortex equilibria,” J. Fluid Mech. 634, 245-268 (2009).

[25] F. J. Blanco-Rodríguez, S. Le Dizès, C. Selçuk, I. Delbende, and M. Rossi, "Internal structure of vortex rings and helical vortices," J. Fluid Mech. 785, 219-247 (2015).

[26] I. Delbende, M. Rossi, and O. Daube, "DNS of flows with helical symmetry," Theoret. Comput. Fluid Dynamics 26, 141-160 (2012).

[27] I. Delbende, B. Piton, and M. Rossi, "Merging of two helical vortices," European Journal of Mechanics-B/Fluids 49, 363-372 (2015).

[28] I. Delbende and M. Rossi, "Dynamics of the three helical vortex system and instability," in Proceedings of the International Conference on Aerodynamics of Offshore Wind Energy Systems and Wakes (ICOWES), Lyngby, Denmark (2013) pp. 224-235.

[29] P. G. Saffman, Vortex dynamics (Cambridge University Press, 1992).

[30] O. Kelbin, A. F. Cheviakov, and M. Oberlack, "New conservation laws of helically symmetric, plane and rotationally symmetric viscous and inviscid flows," J. Fluid Mech. 721, 340-366 (2013).

[31] S. Le Dizès and A. Verga, "Viscous interactions of two co-rotating vortices before merging," J. Fluid Mech. 467, 389-410 (2002)

[32] D. Sipp, L. Jacquin, and C. Cossu, "Self-adaptation and viscous selection in concentrated two-dimensional vortex dipoles," Phys. Fluids 12, 245-248 (2000).

[33] A.J. Callegari and L. Ting, "Motion of a curved vortex filament with decaying vortical core and axial velocity," SIAM J. Appl. Maths 35, 148-175 (1978)

[34] Y. Fukumoto and H. K. Moffatt, "Motion and expansion of a viscous vortex ring. Part 1. A higher-order asymptotic formula for the velocity," J. Fluid Mech. 417, 1-45 (2000).

[35] J. Jiménez, H. K. Moffatt, and C. Vasco, "The structure of the vortices in freely decaying two-dimensional turbulence,” J. Fluid Mech. 313, 209-222 (1996).

[36] S. Le Dizès, "Non-axisymmetric vortices in two-dimensional flows," J. Fluid Mech. 406, 175-198 (2000). 